\title{
Scheduling truck drivers with interdependent routes under European Union regulations
}

\author{
Carlo S. Sartori*, Pieter Smet, Greet Vanden Berghe \\ KU Leuven, Department of Computer Science, CODeS; Leuven.AI, Gebroeders De \\ Smetstraat 1, Gent 9000, Belgium
}

\begin{abstract}
When transporting goods, truck drivers may have to perform load transfers between two vehicles at cross-dock or transfer locations. These transfers create interdependencies between the routes of different truck drivers and require careful temporal synchronization. The situation becomes even more complicated when driver schedules must comply with customer time windows, working periods and regulatory resting requirements. Although the scheduling of hours of service for truck drivers is a well-known problem, existing algorithms cannot cope with interdependent routes. We introduce the Truck Driver Scheduling Problem with Interdependent Routes to successfully accommodate this real-world characteristic. This paper proposes a mathematical model and a label propagation algorithm to solve this new problem under the regulations of the European Union. Experiments indicate that the algorithm can quickly schedule a large number of interdependent routes and outperforms a mathematical programming approach. The algorithm produces valid schedules for multiple drivers and thus becomes a foundation for future vehicle routing algorithms that address interdependent routes and hours of service regulations.
\end{abstract}

Keywords: Scheduling, Truck driver scheduling, Interdependent routes, Hours of service, Label propagation

2010 MSC: 00-01, 99-00

\section{Introduction}

During the daily execution of vehicle routes for logistic operations, the drivers' hours of service (HOS) must comply with certain legal requirements.

\footnotetext{
*Corresponding author. Tel.: +3292658703

Email addresses: carlo.sartori@cs.kuleuven.be (Carlo S. Sartori), pieter.smet@cs.kuleuven.be (Pieter Smet), greet.vandenberghe@cs.kuleuven.be (Greet Vanden Berghe)
} 
For instance, most countries stipulate a maximum time for a driver to continuously operate a vehicle, after which a break must be scheduled. This is not arbitrary, as drivers who do not take breaks for long periods compromise the safety of roads. It is, therefore, crucial to take into account such regulations when developing vehicle routing algorithms.

Additionally, recent increases in e-commerce activity have brought significant challenges to logistic chains, which must deliver products to ever more demanding customers. This increased pressure on transportation companies has motivated the use of cross-docks (Van Belle et al., 2012), or transfer locations (Mitrović-Minić \& Laporte, 2006), where loads are transferred between vehicles to reduce delivery times. Although useful, these operations incur interdependencies between vehicle routes which complicate the development of automated vehicle routing tools.

Goel (2009) introduced the main terminology for the Truck Driver Scheduling Problem (TDSP), which is the name given to the scheduling of routes while respecting HOS regulations. There is no singular definition for the TDSP given that different geographical regions enforce different regulations. The two most widely studied regulations are: the US-TDSP for the United States of America (Goel \& Kok, 2012) and the EU-TDSP for the European Union (Goel, 2009). Despite their differences, all TDSP variants stipulate that drivers must take breaks at regular intervals.

In terms of HOS, we consider the EU regulations. Two basic regulatory guidelines dictate the drivers' HOS. Regulation (EC) No. 561/2006 (European Commission, 2006) concerns driving-related activities. It defines driving periods to be at most 4.5 hours of continuous driving time and defines breaks to be 45-minute intervals which end a driving period. Additionally, the regulation limits the maximum driving time in a day to nine hours. Meanwhile, directive 2002/15/EC (European Commission, 2002) defines rules concerning the accumulated working hours for both driving and service tasks (such as loading and unloading) and limits a working period to at most six continuous hours. A 30-minute break ends a working period. In this research, we consider only a 45-minute break that ends both working and driving periods as in other TDSP-related papers. The time horizon is a single working day for each driver, thus only breaks must be scheduled. Neither split breaks nor rest periods between days are considered.

Our research sets out to explore whether it is possible to extend the current TDSP methods to schedule HOS for drivers with interdependent routes while respecting specific time windows for their tasks. More specifically: is it possible to quickly produce feasible schedules for a set of fixed and interdependent truck routes? To answer this question, this paper introduces the Truck Driver Scheduling Problem with Interdependent Routes (TDSP-IR).

The remainder of this paper is structured as follows. Section 2 reviews the related literature. The TDSP-IR is formally defined in Section 3 together with a mathematical programming formulation. Section 4 introduces a label 
propagation algorithm and its related operations. A complexity analysis of the proposed algorithm is provided in Section 5. Computational results are reported in Section 6, while Section 7 concludes the paper and offers some directions for future research.

\section{Related work}

The literature relevant to the TDSP-IR can be divided into two main areas. One deals with the family of TDSPs, whereas the other considers vehicle routing problems with synchronization constraints. This section explores both of these areas in view of the TDSP-IR challenges.

\subsection{The truck driver scheduling problem}

The TDSP literature is incredibly rich and is typically categorized according to the regulations being considered. Since our paper deals with EU regulations, we refrain from reviewing studies that address other sets of rules, such as the US, Canadian, or Australian regulations. For these cases, the interested reader is referred to the research by Goel \& Kok (2012), Goel \& Rousseau (2012) and Goel et al. (2012).

Goel (2009) introduced the first method, a Label Propagation Algorithm (LPA), to check route feasibility in a week-long horizon that requires the scheduling of both breaks and rest periods between days. Goel (2010) further elaborated on the regulations of the EU-TDSP and introduced a new LPA that either finds a feasible schedule or proves none exists. In both studies, the LPA was devised as a feasibility check for Vehicle Routing Problems (VRPs) to ensure that VRP solutions respect the European Union's regulations.

The two aforementioned approaches assumed that breaks or rests can be taken anywhere en-route. This is not necessarily true as drivers may have to travel for quite some time before finding an appropriate parking space for their vehicle. This could lead to breaching the regulations expressed in (EC) No. 561/2006.

Kok et al. (2011) addressed the scheduling of truck drivers under one working day (time horizon of 13 hours) and time-dependent travel times. Similar to our problem settings, they considered breaks to be restricted to customer locations. They solved the scheduling problem with a MILP formulation and showed that execution times were short for practical uses, even for time-dependent travel times.

Goel (2012) proposed a dynamic programming algorithm to create minimum duration schedules for the EU-TDSP when breaks are limited to customer locations, but no polynomial-time bound on the algorithm's worstcase performance was provided. Goel's dynamic programming approach is actually very similar to LPAs in terms of how solutions are enumerated. 
Kleff (2019) introduced the first polynomial-time algorithm for the EUTDSP when breaks are restricted to customer locations. The proposed algorithm is an LPA, but schedules are primarily represented using piecewise linear functions. This is not a straightforward representation for our problem, despite its interesting results for the single-route TDSP.

More recently, Goel \& Irnich (2017) introduced the first method for VRPs which address both US and EU regulations by means of a branchand-price technique. Meanwhile, Goel (2018) used the proposed exact solver to study how night-time provisions from Directive 2002/15/EC impact the scheduling of truck drivers. Finally, given that producing TDSP-compliant schedules is a computationally cumbersome task when solving the integrated VRP and TDSP problem, Tilk \& Goel (2020) developed a biderectional label propagation method to speed up the schedule generation. They employed this strategy within a branch-and-price-and-cut algorithm to solve the VRP with hours of service regulations and demonstrated the significant advantages of the bidirectional labeling compared to its unidirectional version.

In a broader study, Goel \& Vidal (2013) set out to understand the impact of HOS regulations on VRP solutions. They evaluated route feasibility during heuristic search by implementing LPAs from the literature. In doing so, it was possible to employ one implementation of a VRP metaheuristic that could create a solution compliant with a specific set of regulations by simply selecting the appropriate LPA. Their approach is important because it shows that studying independent scheduling methods for truck drivers enables one to apply those methods in general VRP algorithms, regardless of the type of metaheuristic or solution representation. The scheduling algorithm works as a "plug-and-play" modular component. Indeed, it is the previous success of applying LPAs to solve TDSPs that has motivated us to investigate their applicability to the TDSP-IR.

An overview of the research related to the TDSP is presented in Table1. The main characteristics of the scheduling problem are presented in the columns: the objective (whether the algorithm must generate an optimal or a feasible schedule), the regulation(s) incorporated, the number of time windows per location and whether interdependent routes are considered.

Table 1 reveals some interesting patterns. First, most papers studied the decision problem, rather than an optimization problem (such as minimization of the route duration). Second, research has focused mainly on either the US- or the EU-TDSP. Third, greater attention was given to the simpler case with a single time window per customer location. Finally, no previous research related to the TDSP has considered interdependent routes, even though there are many VRPs which incorporate interdependencies and task synchronization, as we will discuss in the following section. 
Table 1: Summary of TDSP-related research.

\begin{tabular}{|c|c|c|c|c|c|c|c|c|c|}
\hline \multirow[t]{2}{*}{ Reference } & \multicolumn{2}{|c|}{ Objective } & \multicolumn{4}{|c|}{ Regulations } & \multicolumn{2}{|c|}{ Time windows } & \multirow[b]{2}{*}{ Interdep. } \\
\hline & Opt. & Feas. & US & EU & CAN & AUS & Single & Multiple & \\
\hline Goel 2009 & & - & & - & & & - & & \\
\hline Goel $(2010)$ & & 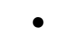 & & - & & & - & & \\
\hline Kok et al. (2011) & - & & & - & & & - & & \\
\hline Goel \& Kok (2012) & & $\bullet$ & - & & & & & - & \\
\hline Goel \& Rousseau (2012) & & - & & & - & & - & & \\
\hline Goel $(2012)$ & - & & - & - & & & & - & \\
\hline Goel et al. (2012) & & - & & & & - & - & & \\
\hline Goel \& Vidal 2013 & & - & - & - & - & - & - & & \\
\hline Goel \& Irnich (2017) & & - & - & - & & & - & & \\
\hline Goel $(2018$ & - & & & - & & & - & & \\
\hline Kleft & $\bullet$ & - & - & - & & & & - & \\
\hline Tilk \& Goel $(2020)$ & & $\bullet$ & - & - & & & - & & \\
\hline This work & & - & & - & & & - & & - \\
\hline
\end{tabular}

\subsection{Vehicle routing problems with task synchronization}

A number of researchers have considered interdependent routes in generalizations of established vehicle routing problems. Dohn et al. (2011) described the five most common types of temporal dependencies between tasks in different routes, thereby leading to problems with interdependent routes. Our paper addresses one of these temporal dependencies, called minimum difference (detailed in Section 3.1).

Drexl (2013) reviewed vehicle routing problems with trailers and transshipment including synchronization constraints. He concluded that only a few papers have examined the interplay of HOS and interdependencies, while none tackled the problem we consider in this paper.

Interdependence constraints have also been studied in transfer or transshipment operations. Mitrović-Minić \& Laporte (2006) introduced the Pickup and Delivery Problem with Transshipment (PDPT). Contrary to usual pickup and delivery problems, the PDPT allows the pickup and the delivery location of a request to be served by different vehicles. An intermediate transfer location may be employed which serves as a temporary storage where vehicles are allowed to drop loads which can then be picked-up later by a second vehicle. A clear interdependence arises here because a vehicle must first unload at a transfer location before another vehicle can pick up the load.

The Vehicle Routing Problem with Cross-Docking (Van Belle et al., 2012) is similar to the PDPT. In this version of the VRP, vehicles must collect products from pickup locations, bring these products together at a crossdock and then deliver those products to another set of customers. Loads may be transferred between vehicles at the cross-dock facility. Similar to the PDPT, synchronization between vehicle routes may be required since vehicles that collect loads at the cross-dock can only leave the facility once all load transferring has finished, thus defining interdependencies between several vehicle routes.

No previous research has considered specialized algorithms to schedule 
truck drivers' HOS in the PDPT or VRP with cross-docking. This is surprising, given that when interdependencies exist, leaving the scheduling of HOS to a post-routing process can lead to significant cascading effects that render all routes infeasible. This is especially the case when there is a tight, limited route duration. In such situations adding a single 45-minute break to a route can cause disruptions, but not adding the mandatory breaks may create legal issues for logistic companies.

Finally, despite some similarities with scheduling problems encountered in airline, railway and bus transportation (Leung, 2004), the HOS regulations for truck transportation are different (Goel \& Vidal, 2013). For example, arrival and working times in road transportation are defined by time windows, whereas the other cases are generally given by a timetable. Therefore, we consider the study of HOS scheduling in VRPs with interdependent routes and the development of fast algorithms for doing so to address a scientific gap of significant practical relevance.

\section{The truck driver scheduling problem with interdependent routes}

This section formally defines the Truck Driver Scheduling Problem with Interdependent Routes and presents a MILP formulation. Examples and characteristics of the TDSP-IR are also discussed.

\subsection{Problem description}

An instance of the TDSP-IR is defined over a set of $m$ fixed vehicle routes. A vehicle route is a sequence of locations $r_{k}=\left(\lambda_{1}, \ldots, \lambda_{\left|r_{k}\right|}\right), k=1, \ldots, m$, where $\left|r_{k}\right|$ denotes the number of locations in route $r_{k}$. These routes are represented as a graph $G=(V, A)$, where $V$ is the set of nodes representing locations, while $A$ is the set of arcs denoting temporal precedence constraints between the nodes in $V$. The parameters of an instance and the decision variables used throughout this paper are summarized in Table 2.

Arc set $A$ is composed of two sets $A=A_{P} \cup A_{R}$ such that $A_{P} \cap A_{R}=\emptyset$. Arc set $A_{R}$ is the set of route arcs of type $(i, j), i, j \in V$, where nodes $i$ and $j$ belong to the same route and node $i$ directly precedes $j$. Each arc has an associated weight $t_{i j}$, which is the travel time from node $i$ to $j$. Meanwhile, set $A_{P}$ contains precedence arcs between nodes in two different routes, that is, they represent interdependence constraints of the type $\left(u_{-}, u_{+}\right), u_{-}, u_{+} \in$ $V$ such that service at node $u_{-}$must end before service at $u_{+}$can begin. These arcs incur the minimum difference constraints (Dohn et al., 2011), which are defined by $H_{u_{-}}+w_{u_{-}} \leq H_{u_{+}}$, where $H_{i}$ is the time at which service begins at node $i \in V$ and $w_{i}$ is the service duration at node $i$.

For an instance to be valid, input graph $G$ must be a directed acyclic graph: arc set $A$ cannot define cycles in $G$. This must always be true because a cycle creates a precedence between at least one node and itself, which can never be resolved (Masson et al., 2013). 
Set $V_{O} \subset V$ contains vertices $o_{k}$ and $d_{k}$, which are the origin and destination nodes of the $k$-th route, respectively. Additionally, every node $i \in V$ has an associated time window $\left[e_{i}, l_{i}\right]$ indicating the earliest time $e_{i}$ and the latest time $l_{i}$ service may begin at this location, as well as a service duration $w_{i}$. If a driver arrives at a location $i$ before $e_{i}$ they are allowed to wait before starting the service at $i$. However, service may never start later than $l_{i}$. For interdependent nodes $\left(u_{-}, u_{+}\right) \in A_{P}$, the start time of service at $u_{+}$also depends on the start time of service at $u_{-}$. Analogously, the latest feasible start time at $u_{-}$may change according to the start time of service at $u_{+}$. Time windows of nodes $o_{k}, d_{k} \in V_{O}$ define the time horizon $\mathcal{H}_{k}$ of each route $r_{k}$, where $e_{o_{k}}$ is the earliest start time and $l_{d_{k}}$ is the latest end time. For simplicity, we will assume the time horizons of all routes are the same and denoted as $\mathcal{H}$.

Table 2: Notation used throughout the paper.

\begin{tabular}{ll}
\hline \multicolumn{2}{l}{ Instance parameters } \\
\hline$n$ & total number of nodes $|V|$ \\
$m$ & total number of routes (or drivers) \\
$i \in V$ & a node to be visited \\
$o_{k}, d_{k} \in V_{O}$ & origin and destination nodes of the $k$-th route \\
$(i, j) \in A_{R}$ & set of route arcs (simple precedence arcs) \\
$\left(u_{-}, u_{+}\right) \in A_{P}$ & set of interdependence arcs $\left(u_{-}, u_{+} \in V\right)$ \\
{$\left[e_{i}, l_{i}\right]$} & time window associated with node $i \in V$ \\
$w_{i}$ & service duration at node $i \in V$ \\
$t_{i j}$ & travel time associated with arc $(i, j) \in A_{R}$ \\
$\mathcal{H}$ & time horizon of all routes \\
$M_{\text {drive }}$ & maximum continuous driving time \\
$M_{\text {work }}$ & maximum continuous working time \\
$M_{\text {daily }}$ & maximum daily driving time \\
$M_{\text {dur }}$ & maximum route duration \\
$B$ & minimum duration of a break period \\
\hline Variables & time at which service begins at node $i$ \\
$H_{i}$ & accumulated continuous driving time up to node $i$ \\
$D_{i}$ & accumulated continuous working time up to node $i$ \\
$W_{i}$ & binary variable which equals 1 when a break is scheduled \\
$x_{i}$ & immediately after service at node $i$ and 0 otherwise \\
\hline
\end{tabular}

Each route $r_{k}$ must be completed by a single truck driver. The driver's schedule must not only comply with customer time windows, but also with HOS regulations. In other words, a break of minimum length $B$ must be scheduled before the driver accumulates $M_{\text {drive }}$ or $M_{\text {work }}$ continuous driving or working time, respectively. A break is only allowed at customer locations after completing the service. However, this could easily be adapted to consider breaks before service begins (Goel, 2012). Once a break has been taken, both accumulated driving and working time are reset to zero and the driver is allowed to drive and work an additional $M_{\text {drive }}$ and $M_{\text {work }}$ units of time. Note that working time includes both driving times and service 
duration at nodes. A route must not exceed the maximum duration $M_{\text {dur }}$, which serves as the shift length of a driver. Finally, $M_{\text {daily }}$ restricts the total driving time in one day. For simplicity, we assume that any route in a TDSP-IR instance does not exceed $M_{\text {daily }}$. In our case, this can be trivially verified in linear time before solving the problem.

The goal of the TDSP-IR is to build a feasible schedule for each route that complies with both customer time windows and HOS regulations. Therefore, a solution method must provide both the departure time for all routes and the breaks during those routes.

To better illustrate the problem, consider the instance in Figure 1 with $m=3$ routes. Arc weights represent travel times while service durations are $w_{i}=0 \mathrm{~h} 30, \forall i \in V$. Drivers can depart from their origin no earlier than 8:00 and must complete their route no later than 21:00. The only time windows relevant in Figure 1 are those at nodes $e$ and $f$. All other time windows are assumed to be [8:00,21:00]. Interdependencies are $\left(a_{-}, a_{+}\right),\left(c_{-}, c_{+}\right) \in A_{P}$.

Let us further define the HOS regulations as those of the EU: $M_{\text {drive }}=4 \mathrm{~h} 30$, $M_{\text {work }}=6 \mathrm{~h}, M_{\text {daily }}=9 \mathrm{~h}, M_{\text {dur }}=10 \mathrm{~h}$ and $B=0 \mathrm{~h} 45$. The length of the time horizon is $13 \mathrm{~h}$. A schedule for each route that complies with time windows, interdependence constraints, $M_{\text {drive }}, M_{\text {work }}$ and $M_{\text {daily }}$ regulations, but which exceeds $M_{\text {dur }}$ for two routes is shown in Figure 2(a). Gray squares denote the service duration at each node, blue rectangles (D) denote driving periods, red rectangles (B) denote scheduled breaks, while white rectangles (I) denote idle or waiting periods. All three schedules begin their routes at time $t=8: 00$, which incurs unnecessary waiting time. As a result, schedules for routes $r_{1}$ and $r_{2}$ are infeasible because they have a total duration of $11 \mathrm{~h} 30>M_{\text {dur }}$ and $11 \mathrm{~h}>M_{\text {dur }}$, respectively. Note that the schedule in $r_{1}$ has unnecessary waiting time at $a_{+}$even though this location has no time window. This is due to the start time of service at $a_{-}$.

Figure 1: A TDSP-IR instance. Solid arcs belong to set $A_{R}$ and dotted $\operatorname{arcs}$ to set $A_{P}$.

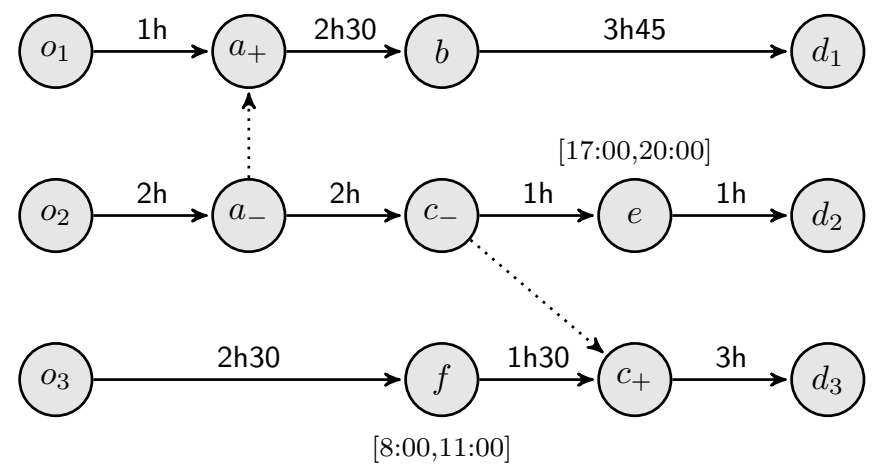


Figure 2: Example of two sets of schedules for a TDSP-IR instance.

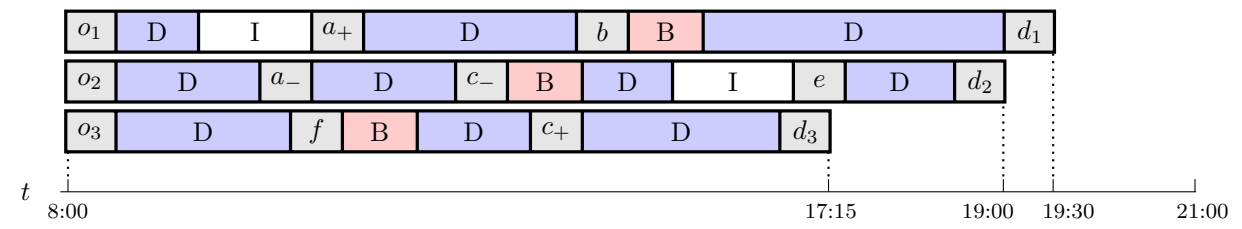

(a) Infeasible schedules beginning at time $t=8: 00$ (some durations exceed $M_{\text {dur }}=10 \mathrm{~h}$ ).

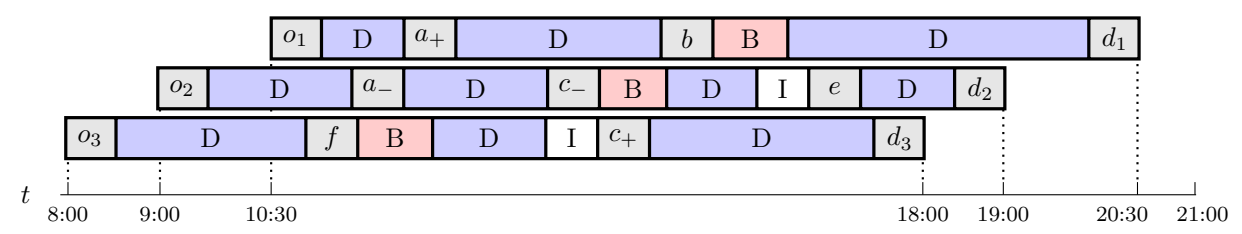

(b) Feasible schedules with postponed departure times (all durations respect $M_{\mathrm{dur}}=10 \mathrm{~h}$ ).

Modifying the start time of $r_{2}$ to reduce the waiting time at $e$ increases the time spent waiting at $a_{+}$and the duration of $r_{1}$ even further. Since route $r_{1}$ has no tight time windows, it is possible to completely remove the idle time by postponing the start time of its service as much as possible. However, waiting in $r_{2}$ cannot be completely eliminated because the time window at $f$ forbids route $r_{3}$ to be postponed. Figure 2(b) presents three feasible schedules with postponed starting times. All three routes have a duration of $10 \mathrm{~h}$.

Throughout the remainder of this paper we will assume that the earliest start time of all routes is $t=0$, which coincides with the beginning of the time horizon. In this way, the latest possible completion time coincides with the length of the time horizon: $\mathcal{H}$. Service time windows are adjusted in accordance with these assumptions.

\subsection{Mathematical programming formulation}

The TDSP-IR can be modeled as a MILP using the variables presented in Table 2, The set of constraints is then written as follows:

$$
\begin{aligned}
H_{j} \geq H_{i}+t_{i j}+w_{i}+x_{i} B, & \forall(i, j) \in A_{R} \\
e_{i} \leq H_{i} \leq l_{i}, & \forall i \in V \\
H_{u_{-}}+w_{u_{-}} \leq H_{u_{+}}, & \forall\left(u_{-}, u_{+}\right) \in A_{P} \\
D_{j} \geq D_{i}+t_{i j}-x_{i} M_{\text {drive }}, & \forall(i, j) \in A_{R} \\
D_{j} \geq t_{i j}, & \forall(i, j) \in A \\
W_{j} \geq W_{i}+t_{i j}+w_{j}-x_{i} M_{\text {work }}, & \forall(i, j) \in A_{R}
\end{aligned}
$$




$$
\begin{aligned}
W_{j} \geq t_{i j}+w_{j}, & \forall(i, j) \in A_{R} \\
0 \leq D_{i} \leq M_{\text {drive }}, & \forall i \in V \\
0 \leq W_{i} \leq M_{\text {work }}, & \forall i \in V \\
H_{d_{k}}-H_{o_{k}} \leq M_{\text {dur }}, & \forall o_{k}, d_{k} \in V_{O} \\
x_{i} \in\{0,1\}, & \forall i \in V
\end{aligned}
$$

Constraints (1) update the start of service variables along a route in accordance with the travel times, service times and breaks. Time window compliance for every customer location is ensured by Constraints (2). Constraints (3) guarantee that for precedence pairs $\left(u_{-}, u_{+}\right) \in A_{P}$, service at node $u_{-}$always ends before service at node $u_{+}$begins. For continuous driving and working time, Constraints (4)-(7) update the values according to the sequence of nodes and are responsible for resetting the corresponding variables whenever a break takes place. Constraints (8) and (9) ensure compliance with maximum driving and working HOS. Constraints (10) guarantee that the length of a route never exceeds the maximum duration. Finally, Constraints (11) restrict $x$ variables to assume only binary values.

Computational experiments indicated that the MILP formulation was too slow for use in iterative algorithms for vehicle routing problems. Therefore, we turned our attention to the development of a specialized approach to solve the TDSP-IR.

\section{Label propagation algorithm}

Based on previous research for the TDSP, we propose a Label Propagation Algorithm (LPA): an iterative technique that propagates labels, here representing a set of partial driver schedules, until a valid solution is either found or proven not to exist. For the single-route TDSP, all iterations propagate labels from one node to the next within the same route. However, in the TDSP-IR, iterations propagate information across multiple routes until all of the routes are scheduled.

This section begins by providing an overview of the algorithm, highlighting its main components. A label is then formally defined in Section 4.2. Operations over labels and their enumeration process are detailed in Sections 4.3 and 4.4 , respectively. Section 4.5 describes the feasibility conditions of labels. Section 4.6 presents an example of the LPA's execution. Finally, dominance criteria are presented in Section 4.7 .

\subsection{Algorithm overview}

An overview of the LPA is shown in Algorithm 1, which receives as input a TDSP-IR instance with graph $G$. The output is a boolean value indicating whether the instance is feasible with respect to all the TDSP-IR constraints. 


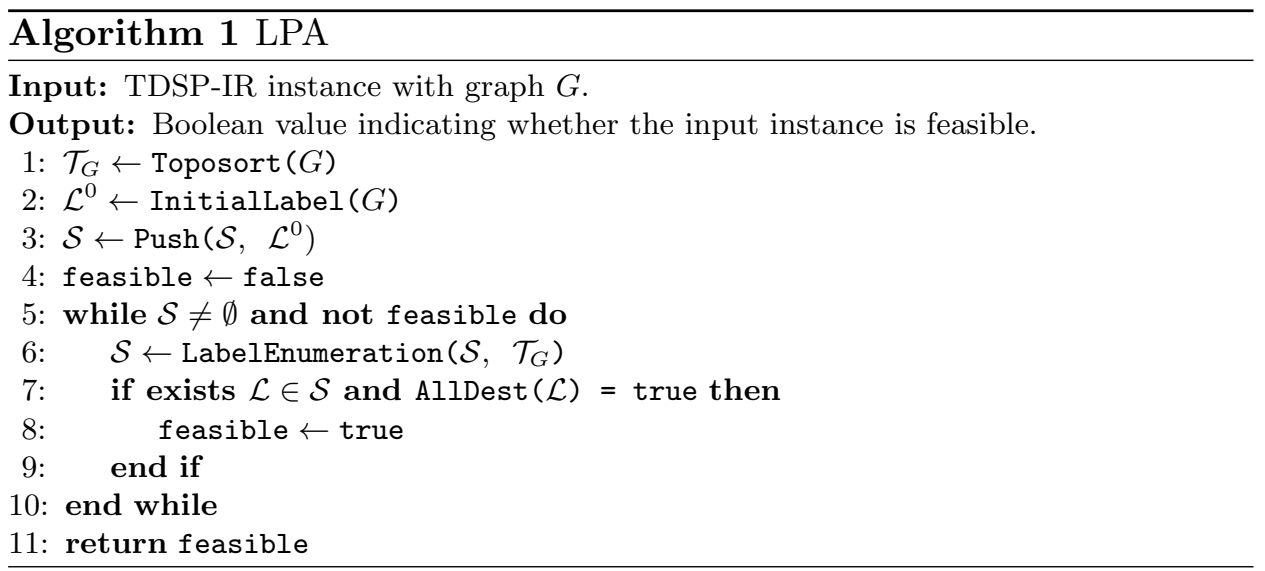

Line 1 generates a topological order $\mathcal{T}_{G}$ (Cormen et al., 2009) of the input graph $G$. This order transforms the propagation across multiple routes into a sequential propagation, as though there was only one route. Such an order is necessary to define the precise sequence of propagations that respects the interdependent tasks in set $A_{P}$. In other words, order $\mathcal{T}_{G}$ ensures that whenever a node $u_{+}$from a pair $\left(u_{-}, u_{+}\right) \in A_{P}$ is to be processed, the corresponding $u_{-}$has already been processed. Since $G$ is a directed acyclic graph, it is always possible to obtain a topological order. An example of this order for the graph in Figure 1 is:

$$
\mathcal{T}_{G}=\left(a_{-}, c_{-}, e, d_{2}, a_{+}, b, d_{1}, f, c_{+}, d_{3}\right)
$$

Note that origin nodes are not included because they are assumed to be the starting location for each route. Order $\mathcal{T}_{G}$ is fixed and decided in this step of the algorithm. Procedure Toposort may be any valid topological sort algorithm, however certain characteristics may improve the performance of the LPA (see Section 4.4).

Line 2 creates an initial label $\mathcal{L}^{0}$, which defines the beginning of the propagation. This label is stored in stack $\mathcal{S}$ by pushing it to the top (line $3)$. The main loop spanning lines 5-10 calls the necessary procedure to propagate labels. Procedure LabelEnumeration generates labels by taking a label from the top of $\mathcal{S}$, propagating it over multiple iterations and storing all the newly generated labels back into $\mathcal{S}$. This procedure is fully described in Section 4.4. Should stack $\mathcal{S}$ become empty during the execution of Algorithm 1, then no feasible solution exists and the procedure terminates indicating infeasibility.

Whenever a valid label has been propagated throughout all nodes in $\mathcal{T}_{G}$, the algorithm terminates (lines 7-9). In other words, once all destination nodes $d_{k}$ have been assigned an earliest feasible starting time of service in a label $\mathcal{L} \in \mathcal{S}$ for all routes $r_{k}, k=1, \ldots, m$, then there is no node in $\mathcal{T}_{G}$ which has remaining successors for the propagation to continue. Hence, 
label $\mathcal{L}$ represents a feasible TDSP-IR solution. This condition is denoted by AllDest $(\mathcal{L})$ in Algorithm 1. Note that the termination condition is similar to the criterion of the label propagation introduced by Goel (2010), which served as a source of inspiration for our own LPA, but we modified the condition to accommodate the interdependent routes being addressed in our study.

\subsection{Label definition}

A label $\mathcal{L}$ stores information for a set of $m$ partial schedules, one for each route in the TDSP-IR. The variables in Table 2 are stored in each label $\mathcal{L}$ and we denote them by $\mathcal{L}\left(H_{i}\right), \mathcal{L}\left(D_{i}\right), \mathcal{L}\left(W_{i}\right), \mathcal{L}\left(x_{i}\right)$ : the earliest feasible start time of service at node $i$, the continuous driving time up until node $i$, the continuous working time up until node $i$ and whether a break is scheduled immediately after servicing node $i$. Recall that nodes are unique and always belong to a single route.

Labels also store slack values $\mathcal{L}\left(S_{k i}\right)$, which correspond to the accumulated waiting time from every origin $o_{k}, \forall k \in \Phi(i)$ up to every node $i$. This is based on the definition of total waiting time offered by Masson et al. (2013). Here, $\Phi(i)$ denotes all the route indices $k$ for which $o_{k}$ precedes node $i$ in $G$. For example, in the graph in Figure 1, $\Phi(e)=\{2\}$ because only $o_{2}$ precedes node $e$. Meanwhile, $\Phi(b)=\{1,2\}$ because $o_{1}$ clearly precedes node $b$, while the path $\left(o_{2}, a_{-}, a_{+}, b\right)$ shows that $o_{2}$ also precedes $b$. The slack is necessary to indicate how much each route in the TDSP-IR can be postponed without affecting any services. The slack is used in the dominance conditions (Section 4.7) to avoid the removal of labels which contain a feasible solution with respect to maximum route duration.

Moreover, labels store push values $\mathcal{L}\left(P_{k}\right)$. They indicate how much the start time of service at $o_{k}, k=1, \ldots, m$, that is $\mathcal{L}\left(H_{o_{k}}\right)$, may be postponed without causing route $r_{k}$, or any other dependent routes, to become infeasible with respect to time windows.

To keep track of the propagation across multiple routes, every label stores values $\mathcal{L}\left(i_{k}\right), k=1, \ldots, m$ denoting the last node scheduled in route $r_{k}$. Meanwhile, $\mathcal{L}(p)$ denotes the next node to be scheduled for this label following the order $\mathcal{T}_{G}$. To clarify, consider the topological order of Figure1: $\mathcal{T}_{G}=\left(a_{-}, c_{-}, e, d_{2}, a_{+}, b, d_{1}, f, c_{+}, d_{3}\right)$. A label with $\mathcal{L}(p)=3$ holds values $\mathcal{L}\left(i_{1}\right)=o_{1}, \mathcal{L}\left(i_{2}\right)=c_{-}$and $\mathcal{L}\left(i_{3}\right)=o_{3}$. Then, $\mathcal{T}_{G}[\mathcal{L}(p)]$ provides the next node to be reached in the propagation of label $\mathcal{L}$ in the linear sequence. Thus, for the example in Figure 1, the next propagation will be from $c_{-}$to $e$ in route $r_{2}$ given that $\mathcal{T}_{G}[\mathcal{L}(p)]=\mathcal{T}_{G}[3]=e$.

The initial label $\mathcal{L}^{0}$ is defined as:

$$
\begin{aligned}
& \mathcal{L}^{0}(p)=1 \\
& \mathcal{L}^{0}\left(i_{k}\right)=o_{k} \text {, } \\
& k=1, \ldots, m
\end{aligned}
$$




$$
\begin{array}{lr}
\mathcal{L}^{0}\left(H_{o_{k}}\right)=\mathcal{L}^{0}\left(D_{o_{k}}\right)=\mathcal{L}^{0}\left(W_{o_{k}}\right)=0, & k=1, \ldots, m \\
\mathcal{L}^{0}\left(S_{k_{k}}\right)=0, & k=1, \ldots, m \\
\mathcal{L}^{0}\left(P_{k}\right)=l_{o_{k}}, & k=1, \ldots, m \\
\mathcal{L}^{0}\left(x_{i}\right)=0, & \forall i \in V
\end{array}
$$

\subsection{Propagation operations}

A propagation refers to a single move from a node $i$ to $j$ in one route $r_{k}$. This one move creates exactly two labels from $\mathcal{L}$. The first, denoted $\mathcal{L}^{\text {direct, }}$

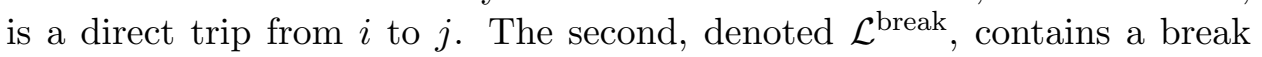
after service at node $i$ before continuing on to $j$. The difference between the two labels is that $\mathcal{L}^{\text {direct }}\left(x_{i}\right)=0$ and $\mathcal{L}^{\text {break }}\left(x_{i}\right)=1$. All the other values in these labels are computed according to standard operations that depend on the value of variable $x_{i}$.

Equations (12) 18 describe the propagation operations. Value $\tilde{e}_{j}$ is the earliest start time of service at $j$. It is $\tilde{e}_{j}=\max \left\{e_{u_{+}}, \mathcal{L}\left(H_{u_{-}}\right)+w_{u_{-}}\right\}$if $j=u_{+}$for $\left(u_{-}, u_{+}\right) \in A_{P}$ and $\tilde{e}_{j}=e_{j}$ otherwise. The first definition is used to guarantee compliance with the interdependence constraints.

$$
\begin{aligned}
\mathcal{L}\left(i_{k}\right) & =j \\
\mathcal{L}(p) & =\mathcal{L}(p)+1 \\
\mathcal{L}\left(H_{j}\right) & =\max \left\{\tilde{e}_{j}, \mathcal{L}\left(H_{i}\right)+w_{i}+\mathcal{L}\left(x_{i}\right) \cdot B+t_{i j}\right\} \\
\mathcal{L}\left(D_{j}\right) & =\mathcal{L}\left(x_{i}\right) \cdot \mathcal{L}\left(D_{i}\right)+t_{i j} \\
\mathcal{L}\left(W_{j}\right) & =\mathcal{L}\left(x_{i}\right) \cdot\left(\mathcal{L}\left(W_{i}\right)+w_{i}\right)+t_{i j}
\end{aligned}
$$

Slack values are also propagated from $i$ to $j$. Due to the interdependencies, slack values propagate from all origins $o_{z}, z \in \Phi(i)$ which precede node $i$ (and necessarily node $j$ ). Slack value $\mathcal{L}\left(S_{z i}\right)$ is propagated from $i$ to $j$ by:

$$
\mathcal{L}\left(S_{z j}\right)=\mathcal{L}\left(S_{z i}\right)+\max \left\{0, \tilde{e}_{j}-\left(\mathcal{L}\left(H_{i}\right)+w_{i}+\mathcal{L}\left(x_{i}\right) \cdot B+t_{i j}\right)\right\}, \forall z \in \Phi(i)
$$

If $j=u_{+}$in a pair $\left(u_{-}, u_{+}\right) \in A_{P}$, then we must also consider the slack from origins that precede the corresponding node $u_{-}$:

$\mathcal{L}\left(S_{z u_{+}}\right)=\min \left\{\mathcal{L}\left(S_{z u_{+}}\right), \mathcal{L}\left(S_{z u_{-}}\right)+\max \left\{0, \mathcal{L}\left(H_{u_{+}}\right)-\left(\mathcal{L}\left(H_{u_{-}}\right)+w_{u_{-}}\right)\right\}\right\}, \forall z \in \Phi\left(u_{-}\right)$

Meanwhile, the push value is updated for each route where the origin precedes $j$ by:

$$
\mathcal{L}\left(P_{z}\right)=\min \left\{\mathcal{L}\left(P_{z}\right), \mathcal{L}\left(S_{z j}\right)+l_{j}-\mathcal{L}\left(H_{j}\right)\right\}, \forall z \in \Phi(j)
$$

One label propagation has time complexity $\mathcal{O}(m)$ since it must consider all $z \in \Phi(j)$, which can contain at most $m$ elements. The memory con- 
sumption of a label depends on both $n$ and $m$. Variables $\mathcal{L}\left(H_{i}\right), \mathcal{L}\left(D_{i}\right)$, $\mathcal{L}\left(W_{i}\right)$ and $\mathcal{L}\left(x_{i}\right)$ are stored for every node in $G$, thus requiring $\mathcal{O}(n)$ memory. Meanwhile $\mathcal{L}\left(P_{k}\right)$ requires $\mathcal{O}(m)$ memory and $\mathcal{L}\left(S_{k i}\right)$ requires $\mathcal{O}(n m)$ memory. Hence a label requires $\mathcal{O}(\mathrm{nm})$ memory.

\subsection{Label enumeration}

Algorithm 2 describes the procedure LabelEnumeration used in Algorithm 1 to generate labels, that is, to propagate them iteratively. This function is the main component of the LPA in terms of enumerating solutions. It begins by taking one label from the top of stack $\mathcal{S}$ and pushing it to queue $\mathcal{Q}$. In line $2, r(i)$ provides the index of the route to which node $i$ belongs: an index $k$ in the range $1, \ldots, m$. The value of $k$ is constant throughout the execution of a single call to Algorithm 2 from the main loop in Algorithm 1. Indeed, once Algorithm 2 detects that further propagations change the value of $k$, it begins the final steps of the current call by pushing labels from $\mathcal{Q}$ to $\mathcal{S}$ (line 12), effectively emptying the queue. This ensures that each call to the procedure only propagates labels for node sequences in the same route $r_{k}$. Hence, the label enumeration procedure is repeated for every $k$, sometimes even more than once, in order to obtain a solution.

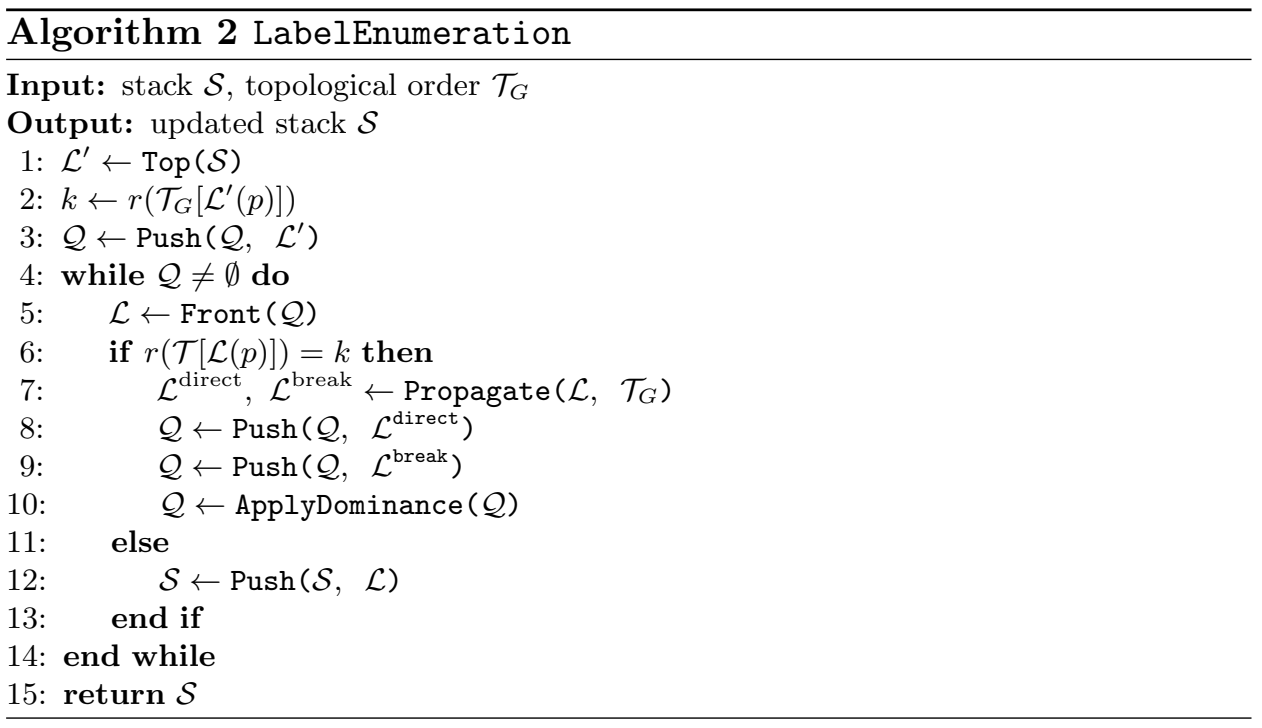

The main loop of the algorithm (lines 4-14) is repeated so long as there are labels in $\mathcal{Q}$. In each iteration, a label $\mathcal{L}$ is removed from the queue. Line 6 checks whether the next propagation of $\mathcal{L}$ considers the same route $r_{k}$ as the previous ones. If this is true, then two labels $\mathcal{L}^{\text {direct }}$ and $\mathcal{L}^{\text {break }}$ are created from $\mathcal{L}$ in accordance with the operations described in Section 4.3 . Both labels are pushed to the queue (lines 8-9) followed by a dominance test (explained in Section 4.7) to remove redundant labels from $\mathcal{Q}$ (line 10). However, if a route which differs from $r_{k}$ would be modified in the next 
propagation, then label $\mathcal{L}$ is added to stack $\mathcal{S}$ instead of being propagated (line 12). Once $\mathcal{Q}$ becomes empty, the algorithm terminates and returns $\mathcal{S}$. Note that a label is only pushed to either $\mathcal{Q}$ (lines 8-9) or $\mathcal{S}$ (line 12) if it is feasible (see Section 4.5). An infeasible or dominated label is always excluded from the propagation.

While Algorithm 1 applies Depth-First Search (DFS) by employing a stack, Algorithm 2 applies Breadth-First Search (BFS) by employing a queue. The idea is to benefit from the problem structure, trying to propagate labels in one route using a BFS (Algorithm 2), which has been observed to perform better than its DFS-counterpart (Goel, 2010). On the other hand, our preliminary experiments showed that a DFS strategy performed better when moving across routes in the propagation by decreasing memory usage when solving large problem instances. Section 4.7 shows how this DFS-BFS search strategy reduces the complexity of dominance tests.

The performance of this search strategy is, however, highly dependent on the structure of the topological order. The order in $\mathcal{T}_{G}$ should contain sequences of nodes belonging to the same route that are as long as possible in order to benefit from the BFS phase (lines $5-10$ ). Otherwise, if neighboring nodes in $\mathcal{T}_{G}$ always belong to a different route, then the LPA becomes a full DFS search, which tends to be inefficient for large instances. For this reason, we describe how to obtain an efficient topological order in Appendix A.

None of the aforementioned measures reduces the theoretical complexity of the LPA, however. They were simply observed to perform well.

\subsection{Feasibility conditions}

A label is considered feasible if it respects a number of conditions. Three of these are Conditions (20)-(22), which must be respected for all route indices $k=1, \ldots, m$ considering their last scheduled node $\mathcal{L}\left(i_{k}\right)$, which we will refer to as $i_{k}$ for simplicity.

$$
\begin{aligned}
& \mathcal{L}\left(H_{i_{k}}\right) \leq l_{i_{k}} \\
& \mathcal{L}\left(D_{i_{k}}\right) \leq M_{\text {drive }} \\
& \mathcal{L}\left(W_{i_{k}}\right) \leq M_{\text {work }}
\end{aligned}
$$

However, these conditions do not guarantee compliance with maximum route duration. Unfortunately, the presence of interdependencies makes the computation of route duration nontrivial. To guarantee compliance with maximum route duration constraints, we associate every label $\mathcal{L}$ with a Simple Temporal Problem (STP, Dechter et al. (1991)). In the STP, a set of variables represents the moment in time when a given action takes place. An auxiliary variable is created to represent the origin of the time horizon $t=0$. For simplicity, we will refer to this special variable as $\alpha$. For the TDSP-IR, we associate a variable $h_{i}$ with every node $i \in V$ and variable $h_{\alpha}=0$ with the beginning of the time horizon. 
Let us denote the $\mathrm{STP}$ associated with label $\mathcal{L}$ as $\operatorname{STP}(\mathcal{L})$. A solution to $\operatorname{STP}(\mathcal{L})$ provides valid start times for all drivers for a given TDSP-IR instance. If no solution can be found, the (partial) schedules in $\mathcal{L}$ are infeasible with respect to maximum route duration. Based on Masson et al. (2014), we define the associated $\operatorname{STP}(\mathcal{L})$ as:

$$
\begin{aligned}
h_{i}-h_{j} \leq-w_{i}-t_{i j}-\mathcal{L}\left(x_{i}\right) \cdot B, & \forall(i, j) \in A_{R} \\
h_{u_{-}}-h_{u_{+}} \leq-w_{u_{-}}, & \forall\left(u_{-}, u_{+}\right) \in A_{P} \\
h_{\alpha}-h_{i} \leq-e_{i}, & \forall i \in V \\
h_{i}-h_{\alpha} \leq l_{i}, & \forall i \in V \\
h_{d_{k}}-h_{o_{k}} \leq M_{\mathrm{dur}}, & \forall o_{k}, d_{k} \in V_{O}
\end{aligned}
$$

Due to break scheduling, every label produces a unique STP by means of Constraint 23). Dechter et al. (1991) showed that every STP can be written as a distance graph, where vertices represent the $h_{i}$ variables and where arcs represent the constraints between these variables in the formulation. Note that Constraints (25)-(27) are responsible for creating cycles in the distance graph. Since some arcs have negative weights, the graph may contain negative-cost cycles. Dechter et al. (1991) showed that the STP can be solved by employing a shortest path algorithm that accommodates negative cycles. For example, the Bellman-Ford algorithm leads to a time complexity of $\mathcal{O}\left(n^{2}\right)$, where $n$ is the number of nodes in the TDSP-IR instance. Additionally, the STP has two important characteristics: (i) if there is a negative cycle then the problem is infeasible and (ii) if there is no negative cycle, then the STP is called consistent and its shortest paths provide a valid schedule. For the TDSP-IR, the resulting shortest distances in a consistent STP create valid starting times for all truck drivers' routes.

In summary, a label $\mathcal{L}$ is feasible if it respects Constraints $20-22$ and $\operatorname{STP}(\mathcal{L})$ is consistent. This is ensured for every new label in the LPA to avoid propagating partial solutions which may already be proven infeasible. However, a complete TDSP-IR solution is only obtained once a label $\mathcal{L}$ reaches the last node in $\mathcal{T}_{G}$, in which case the starting time of service in each location is set as $\mathcal{L}\left(H_{i}\right)=h_{i}, \forall i \in V$.

\subsection{An example of the label propagation}

The example in Figure 3 illustrates the execution of the algorithm, the feasibility conditions and the interdependent routes. Suppose values $M_{\text {dur }}=$ 16, $M_{\text {drive }}=7$ and $B=1$. For simplicity, working time is ignored. Let us assume the propagation order of the LPA is $\mathcal{T}_{G}=\left(a_{-}, b, d_{1}, a_{+}, c, d_{2}\right)$. Figure 4 also depicts the execution of the label propagation using the proposed LPA by showing the labels relative to the two routes at every iteration.

Breaks at the origin nodes have no effect on the solution, so these are ignored. The label $\mathcal{L}^{0}$ starting in $o_{1}$ and $o_{2}$ (Figure $\left.4(\mathrm{a})\right)$ is propagated to $a_{-}$ 
following the order $\mathcal{T}_{G}$, generating one label $\mathcal{L}$ which arrives at $\mathcal{L}\left(H_{a_{-}}\right)=5$ (Figure 4(b)). A break must then be scheduled after $a_{-}$since a direct trip from $a_{-}$to $b$ would exceed the maximum continuous driving time $M_{\text {drive }}$. This results in a single feasible label reaching node $b$ at $\mathcal{L}\left(H_{b}\right)=20$ (Figure 4(c)). Note the required waiting time enforced by the time window in $b$. Then, two labels would reach destination $d_{1}$, one with a break at $a_{-}$and another with an additional break at $b$ (Figure $4(\mathrm{~d})$ ). For simplicity, we will ignore the second label because it has no impact on our analysis. This leads to a single label $\mathcal{L}$ reaching $d_{1}$ at time $\mathcal{L}\left(H_{d_{1}}\right)=21$, break $\mathcal{L}\left(x_{a_{-}}\right)=1$, nodes $\mathcal{L}\left(i_{1}\right)=d_{1}, \mathcal{L}\left(i_{2}\right)=o_{2}$ and position $\mathcal{L}(p)=4$. Thus far $\operatorname{STP}(\mathcal{L})$ remains consistent because route $r_{1}$ can be postponed up to $\mathcal{L}\left(H_{o_{1}}\right)=5$, making its total duration $\mathcal{L}\left(H_{d_{1}}\right)-\mathcal{L}\left(H_{o_{1}}\right)=16=M_{\text {dur }}$.

Figure 3: A simple TDSP-IR instance with two routes.

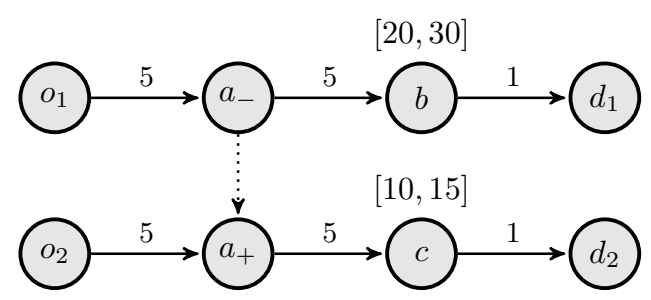

Next, by following the topological order, $\mathcal{T}_{G}[\mathcal{L}(p)]=a_{+}$, the propagation begins in route $r_{2}$ (Figure $4(\mathrm{e})$ ). At this point in Algorithm 2, label $\mathcal{L}$ would be pushed to $\mathcal{S}$ (line 12), returning to Algorithm 1 where a new call to LabelEnumeration would be made to propagate in $r_{2}$.

One label reaches $a_{+}$with $\mathcal{L}\left(H_{a_{+}}\right)=5$ (note that this label is simply the propagation of the label upon $d_{1}$ in $r_{2}$ ). Once again, a break is needed at $a_{+}$for feasibility reasons, resulting in a single label $\mathcal{L}$ reaching node $c$ at $\mathcal{L}\left(H_{c}\right)=11$ (Figure $4(\mathrm{f})$ ). Although this label might initially appear feasible, $\operatorname{STP}(\mathcal{L})$ is not consistent. This is due to the increase in elapsed time from $a_{+}$to $c$ which restricts the start time of service in route $r_{1}$ to be at most $\mathcal{L}\left(H_{O_{1}}\right)=4$, so that service at $c$ begins within the time window. However, the duration of $r_{1}$ then is at least $\mathcal{L}\left(H_{d_{1}}\right)-\mathcal{L}\left(H_{o_{1}}\right)=17>M_{\text {dur }}$ and thus an infeasible label.

In conclusion, the instance depicted in Figure 3 has no solution, but this is not immediately obvious given that it depends not only on the interaction between the two routes but also on the breaks that are scheduled in each route. As the number of nodes, routes and interdependencies increase, it becomes more challenging to decide the feasibility of an instance, thus requiring a dedicated solution method such as the proposed LPA. 
Figure 4: How labels propagate across the routes in the graph of Figure 3 The dashed purple line represents one label and its position with regard to each of the routes after every propagation.

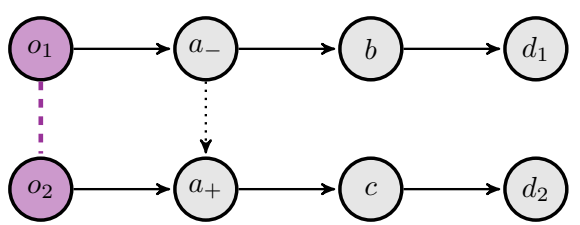

(a) The algorithm starts with $\mathcal{L}^{0}$ at the two origin locations: $o_{1}$ and $o_{2}$ respectively in routes $r_{1}$ and $r_{2}$.

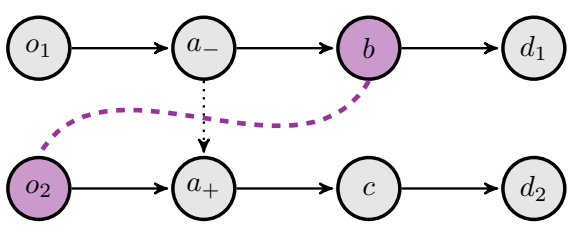

(c) A new propagation in route $r_{1}$ again modifies the label for this route only. For $r_{2}$, the label has not been changed yet.

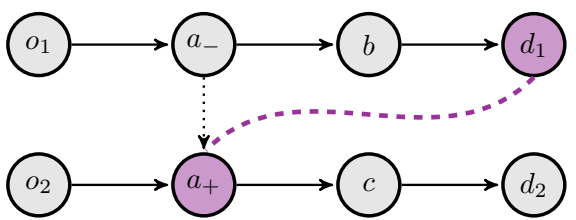

(e) The propagation in route $r_{2}$ begins, following the order $\mathcal{T}_{G}$.

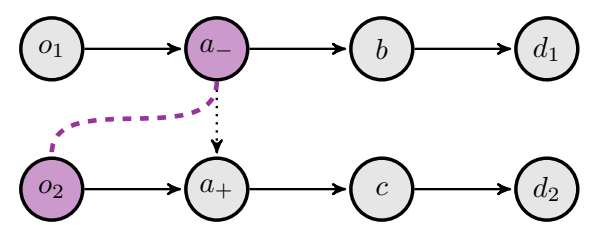

(b) By following $\mathcal{T}_{G}$, the first propagation changes the label position relative to only one route.

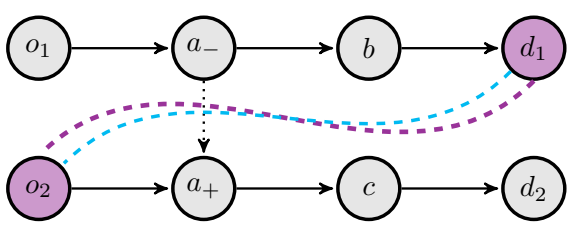

(d) Here, the blue line represents the second label that would be created in the propagation (but for simplicity purposes will be ignored).

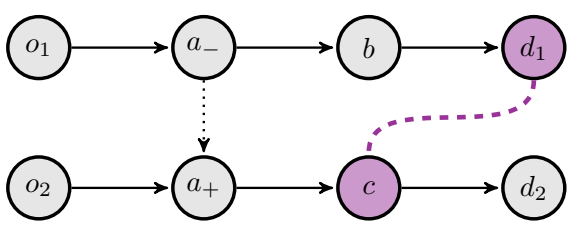

(f) The propagation reaches node $c$ and proves the instance infeasible.

\subsection{Dominance conditions}

A label $\mathcal{L}$ dominates another label $\mathcal{L}^{\prime}$ if Conditions (28)-(34) are satisfied. A precondition to decide dominance is that both labels must be propagated up to the same node in $\mathcal{T}_{G}$. In other words, $\mathcal{L}(p)=\mathcal{L}^{\prime}(p)$. This implies $\mathcal{L}\left(i_{k}\right)=\mathcal{L}^{\prime}\left(i_{k}\right), \quad k=1, \ldots, m$. For simplicity purposes, throughout the remainder of this section we will adopt $i_{k}$ to denote the last scheduled node in route $r_{k}$ for either $\mathcal{L}$ or $\mathcal{L}^{\prime}$. All conditions must simultaneously be true for all routes $r_{k}, k=1, \ldots, m$.

$$
\begin{aligned}
& \mathcal{L}\left(H_{i_{k}}\right) \leq \mathcal{L}^{\prime}\left(H_{i_{k}}\right) \\
& \mathcal{L}\left(D_{i_{k}}\right) \leq \mathcal{L}^{\prime}\left(D_{i_{k}}\right)
\end{aligned}
$$




$$
\begin{aligned}
\mathcal{L}\left(W_{i_{k}}\right) & \leq \mathcal{L}^{\prime}\left(W_{i_{k}}\right) \\
\mathcal{L}\left(P_{k}\right) & \geq \mathcal{L}^{\prime}\left(P_{k}\right) \\
\mathcal{L}\left(S_{z i_{k}}\right) & \geq \mathcal{L}^{\prime}\left(S_{z i_{k}}\right), \quad \forall z \in \Phi\left(i_{k}\right) \\
\mathcal{L}\left(H_{u_{-}}\right) & \leq \mathcal{L}^{\prime}\left(H_{u_{-}}\right), \quad \forall u_{-} \in U_{-}(\mathcal{L}) \\
\mathcal{L}\left(S_{z u_{-}}\right) & \geq \mathcal{L}^{\prime}\left(S_{z u_{-}}\right), \quad \forall u_{-} \in U_{-}(\mathcal{L}), \quad z \in \Phi\left(u_{-}\right)
\end{aligned}
$$

where $\Phi(i)$ is the set defined in Section 4.2. Meanwhile, $U_{-}(\mathcal{L})$ is the set of all $u_{-}$locations that belong to a precedence pair $\left(u_{-}, u_{+}\right) \in A_{P}$ for which $u_{-}$has been scheduled in the partial solution $\mathcal{L}$, while $u_{+}$has not been scheduled. When considering the example in Figure 3, $U_{-}\left(\mathcal{L}^{0}\right)=\emptyset$ for the initial label, but after propagating to $a_{-}$it becomes $U_{-}(\mathcal{L})=\left\{a_{-}\right\}$ and remains like this until $a_{+}$is processed, whereupon the set once again becomes $U_{-}(\mathcal{L})=\emptyset$.

Conditions (28)-(31) are similar to the conditions for the TDSP (Goel, 2010). Condition (32) verifies slack with respect to all origins $o_{z}, z \in \Phi\left(i_{k}\right)$ that precede node $i_{k}$. This ensures that any postponement of $r_{z}$ 's start time has at most as much impact on the service time at $i_{k}$ in $\mathcal{L}$ as in $\mathcal{L}^{\prime}$.

Conditions (33) and (34) compare the values of labels at interdependencies which have not been fully scheduled yet. The intuition behind Condition (33) is that arriving earlier at $u_{-}$provides a greater degree of freedom to (i) postpone service at $u_{-}$without impacting $u_{+}$(considering Equation 12 ) and (ii) choose a start time for service at $u_{+}$without incurring additional waiting time at $u_{+}$'s route (considering Equation 13). Meanwhile, Condition (34) ensures that slack up to the interdependence points in $\mathcal{L}$ allow for a greater degree of freedom than in $\mathcal{L}^{\prime}$ to postpone a route's starting time of service without affecting other routes connected together by these interdependence points. Conditions (33) and (34) are both used to avoid the removal of feasible solutions such as those illustrated in the examples in Appendices B.1 and B.2.

To improve efficiency, once a route $r_{k}$ has been fully scheduled $\left(\mathcal{L}\left(i_{k}\right)=d_{k}\right)$ we may drop Conditions (29) and (30) for route $r_{k}$. This is always true because Conditions 20-22 ensure label $\mathcal{L}$ is feasible in terms of working and driving time for route $r_{k}$. Since $\mathcal{L}\left(i_{k}\right)=d_{k}$, no further propagations will be performed in $r_{k}$ and so values $\mathcal{L}\left(D_{i_{k}}\right)$ and $\mathcal{L}\left(W_{i_{k}}\right)$ will remain fixed. Thus, it is safe to drop Conditions (29) and (30) from the dominance tests for route $r_{k}$.

Then, Conditions (28)-(34) define label dominance. This is formally stated in Lemma 1.

Lemma 1. Given two labels $\mathcal{L}$ and $\mathcal{L}^{\prime}$ with partial schedules for which Conditions (28)-(34) are valid, then either $\mathcal{L}$ produces a feasible solution or neither $\mathcal{L}$ nor $\mathcal{L}^{\prime}$ can produce a feasible solution.

Proof. Appendix C provides the proof. 
Finally, we will verify the asymptotic time complexity of the dominance test. Conditions (28)-(31) require a constant number of operations for each route index $k$, thus $\mathcal{O}(m)$ operations in total. Conditions (32), (33) and (34) require $\mathcal{O}\left(m^{2}\right), \mathcal{O}\left(\left|A_{P}\right|\right)$ and $\mathcal{O}\left(m\left|A_{P}\right|\right)$ operations, respectively. Therefore, a single dominance test has time complexity $\mathcal{O}\left(m^{2}+m\left|A_{P}\right|\right)$. In the proposed LPA, dominance tests are only applied in Algorithm 2. This allows the quadratic term $\mathcal{O}\left(\mathrm{m}^{2}\right)$ in Condition 32 to be reduced to $\mathcal{O}(m)$ since only slack values from origins $o_{z}$ which precede nodes in $r_{k}$ are changed in a call to Algorithm 2. Slack values to any nodes in routes $r_{z}, r_{z} \neq r_{k}$ remain unchanged for all labels during one procedure call and do not need to be tested. The complexity of the dominance test reduces to $\mathcal{O}\left(m\left|A_{P}\right|\right)$.

\subsection{Addressing United States regulations}

A natural question one might ask is whether the proposed LPA can be modified to produce schedules under United States regulations. To recall our literature review, generalizing an algorithm to address both EU and US regulations has been shown to be possible for the single-route TDSP by Goel (2012), Goel \& Irnich (2017) and Kleff (2019).

However, when interdependent routes are considered, adapting the proposed LPA to address US regulations is far from trivial. After the 2013 revision of the US regulations (Goel, 2014), truck drivers must take a break of at least 30 minutes before no more than $8 \mathrm{~h}$ have elapsed since the end of their last break or rest period. While under EU regulations the limitations concerning continuous driving and working times depend only on constant values for driving and working time, in the US the elapsed time also depends on the total waiting time between two breaks. This seemingly innocuous regulatory change leads to two main challenges for the introduced LPA. First, in order to comply with the maximum elapsed time it may be necessary to increase the length of a break beyond the 30-minute minimum, which is never necessary in the EU during a single working day. Second, the elapsed time between two breaks depends on decisions taken in other routes because of necessary waiting times at interdependent locations. This means that it is very unclear how exactly we should specify dominance criteria in order to distinguish between good and bad partial solutions. Given that both of these challenges are non-trivial to address we have included further details in Appendix D, which future researchers may draw inspiration from.

\section{Complexity analysis}

In this section, we turn our attention towards analyzing the worst-case time complexity of the LPA. We begin by presenting an analysis for the general definition of the TDSP-IR in Section 5.1. Then, in Section 5.2, we 
show how the complexity of the LPA may be reduced by dropping a single constraint from the original problem.

\subsection{Complexity of the algorithm}

For the full time complexity of the LPA, we first note that a topological sort may be obtained in strictly polynomial time (Cormen et al., 2009). Since other operations are asymptotically worse, we ignore the complexity of the sorting throughout the remainder of the analysis.

It is not clear how many labels can be dominated overall, or even in each single propagation. The presence of multiple interdependencies makes this analysis nontrivial. Thus, the best bound is the trivial one which considers the worst case: that no label is dominated and all labels must be considered while still comparing them all with one another to verify dominance.

There may be $\mathcal{O}\left(2^{n}\right)$ possible solutions for the TDSP-IR. Every propagation takes $\mathcal{O}(m)$ time and an STP consistency check takes $\mathcal{O}\left(n^{2}\right)$, thus in total $\mathcal{O}\left(2^{n}\left(m+n^{2}\right)\right)$. We may have to compare each solution against all other solutions every iteration to verify dominance, which requires $\mathcal{O}\left(2^{2 n}\right)$ dominance tests. Each dominance test takes $\mathcal{O}\left(m\left|A_{P}\right|\right)$ using the DFS-BFS propagation scheme, resulting in $\mathcal{O}\left(2^{2 n} m\left|A_{P}\right|\right)$. Hence, considering all steps, the asymptotic complexity of the LPA is $\mathcal{O}\left(2^{n}\left(m+n^{2}\right)+4^{n} m\left|A_{P}\right|\right)$, which can be simplified to $\mathcal{O}\left(4^{n} m\left|A_{P}\right|\right)$. Since $n \geq\left|A_{P}\right| \geq m-1$, we may also denote the LPA's final complexity as $\mathcal{O}\left(4^{n} n^{2}\right)$.

\subsection{Complexity when disregarding maximum route duration}

Abandoning compliance with maximum route duration may be interesting for applications where drivers are allowed to work for long shifts. For example, if all routes are performed within a time horizon of $\mathcal{H}=13 \mathrm{~h}(\mathrm{Kok}$ et al., 2011; Goel, 2012) and the maximum duration permitted per route is $M_{\text {dur }}=13 \mathrm{~h}$, then the LPA only needs to decide where to schedule the breaks in each route to comply with customer time windows and interdependencies.

In this case, setting departure times at origin locations to $H_{o_{k}}=0$ is always feasible. Variables $\mathcal{L}\left(S_{k i}\right)$ and $\mathcal{L}\left(P_{k}\right)$ must be verified in dominance tests due to the presence of maximum route duration constraints. When these constraints can be ignored, it is possible to remove Conditions (31), (32) and (34) from the dominance tests.

The impact of this change cannot be underestimated. Eliminating the aforementioned conditions enables one to turn each label propagation into an $\mathcal{O}(1)$ time operation. Label dominance is reduced to verifying Conditions (28)-(30) and (33) with a total complexity of $\mathcal{O}\left(m+\left|A_{P}\right|\right)$. The structure of the LPA further reduces this complexity to $\mathcal{O}\left(\left|A_{P}\right|\right)$ (by applying dominance only in Algorithm 2). Additionally, it is not necessary to verify consistency with the STP, since no duration exists. The overall complexity is thus reduced to $\mathcal{O}\left(4^{n}\left|A_{P}\right|\right)$. 


\section{Computational experiments}

Computational experiments are devised to evaluate the scalability of the LPA. Since there are no TDSP-IR instances available, we generated instances using an insertion heuristic for the PDPT executed over modified instances from Sampaio et al. (2020). The modifications are detailed in Appendix E.1. The resulting TDSP-IR instances had characteristics which lie within the following ranges: $4 \leq n \leq 300,1 \leq m \leq 22$ and $0 \leq\left|A_{P}\right| \leq 34$.

In accordance with $\mathrm{EU}$ regulations, parameter values are set as follows: $M_{\text {drive }}=4 \mathrm{~h} 30, M_{\text {work }}=6 \mathrm{~h} 00, M_{\text {daily }}=9 \mathrm{~h} 00$ and $B=0 \mathrm{~h} 45$. The time horizon $\mathcal{H}=13 \mathrm{~h}$ is the same across all instances and all routes. To show the impact of maximum route duration when solving the problem, we ran experiments varying the shift length of the truck drivers so that they were assigned a short shift of $M_{\mathrm{dur}}=8 \mathrm{~h}$, a medium shift of $M_{\mathrm{dur}}=10 \mathrm{~h}$ or a long shift of $M_{\text {dur }}=13 \mathrm{~h}$ (or no maximum duration).

Results of the LPA are compared against those of the MILP formulation, which was executed without an objective function and seeks only to generate a feasible schedule. The entire approach was implemented in C++ and compiled using GNU g++ version 7.5 in an Ubuntu 20.04 LTS operating system. Experiments were carried out on an Intel Xeon E5-2660 at $2.6 \mathrm{GHz}$ with 160 GB of RAM. The MILP was implemented using the C++ API of Gurobi 9 with default settings and single-thread mode.

First, we inspect the performance of the algorithms over all instances in Section 6.1. The results considering only feasible TDSP-IR instances are reported in Section 6.2. Finally, an analysis of a naive algorithm's performance is presented in Section 6.3 .

\subsection{Performance over all instances}

Figure 5 illustrates the execution times in milliseconds (ms) for both the LPA and the MILP for each shift length. The execution times are the average per run according to the total number of interdependencies in a TDSP-IR instance: the cardinality of set $A_{P}$, which influences many of the asymptotic operation complexities for the LPA and is directly related to the number of routes and nodes (see Appendix E.2). Over all PDPT instances, a total of 89 million executions of the LPA and MILP were performed. Appendix E.3 provides detailed results for these experiments.

The experiments indicate that the LPA almost always outperforms the MILP. This is particularly true for instances with $\left|A_{P}\right| \leq 25$, for which differences in average computation times significantly favor the LPA. For instances with $\left|A_{P}\right| \geq 25$ the execution times present a greater variation for the LPA, often incurring an increase in average computation times. This is not surprising, since an increase in the cardinality of set $A_{P}$ is associated with an increase in the number of nodes or the number of routes in the TDSP-IR instance. 
Figure 5: Average computation times of the LPA and MILP.

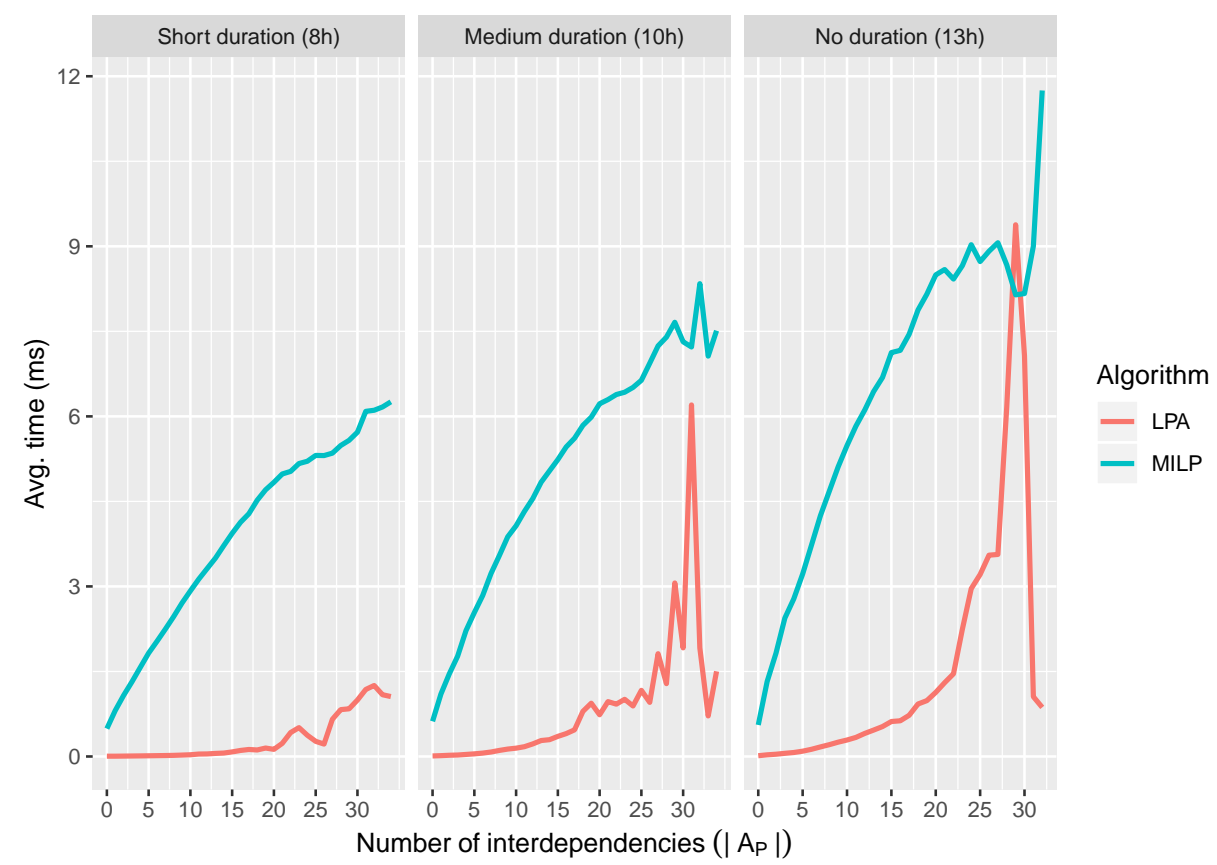

Additionally, varying the maximum route duration has an impact on the average execution time. Both the MILP and LPA require more time to solve TDSP-IR instances as the maximum duration increases. At first, this may seem counter-intuitive given that we showed in Section 5.2 that the worst-case complexity of the LPA reduces when the duration constraints are dropped. However, the number of feasible positions to schedule breaks increases with duration, since routes become less temporally constrained. Similarly, the number of nodes that can feasibly fit into a TDSP-IR instance is larger, further increasing the number of possible break locations and therefore the difficulty of solving these instances.

In terms of total execution time to solve all 89 million instances, the LPA required 129 minutes, while the MILP took 2522 minutes - almost 20 times longer than the LPA. This further confirms that if the purpose of these scheduling algorithms is to iteratively solve multiple instances of the TDSP-IR in a VRP heuristic similar to (Goel \& Vidal, 2013), using the LPA will provide a major advantage concerning the VRP heuristic's observed computation time when compared to using the MILP approach.

Even though we report results for instances with $\left|A_{P}\right| \geq 25$, we should consider how realistic instances containing multiple routes connected by $25-$ 30 interdependent tasks are. This is not only a theoretical challenge, but one of major practical consequences given that any delay or disruption with respect to one customer's service may initiate significant cascading effects 
that render all routes infeasible. Indeed, our own preliminary experiments with real-world data have shown that a Belgian transportation company rarely has more than six routes connected by $\left|A_{P}\right| \leq 10$ interdependencies.

In summary, for applications that impose a limit of $\left|A_{P}\right| \leq 25$ (for practical reasons), our results show the LPA is a safe choice. However, even when this limit may not exist the LPA is expected to present better runtime performance on average than the MILP approach.

\subsection{Performance over feasible instances}

Some intriguing behavior is encountered when exploring the performance of the two algorithms for only the feasible TDSP-IR instances. The average execution time for these instances is shown in Figure 6. The LPA always requires substantially shorter computation times to prove the feasibility compared to proving the infeasibility of an instance. By contrast, the MILP demonstrates the opposite behavior. As a result, the execution time of the LPA exhibits slower growth, while execution times for the MILP exhibit a more steep growth compared to those in Figure 5. Moreover, the graphs show that route duration has less of an impact on the performance for feasible TDSP-IR instances, although some variation can still be observed. Appendix E.3 provides detailed results for these experiments.

Figure 6: Average execution times of the LPA and MILP for feasible TDSP-IR instances.

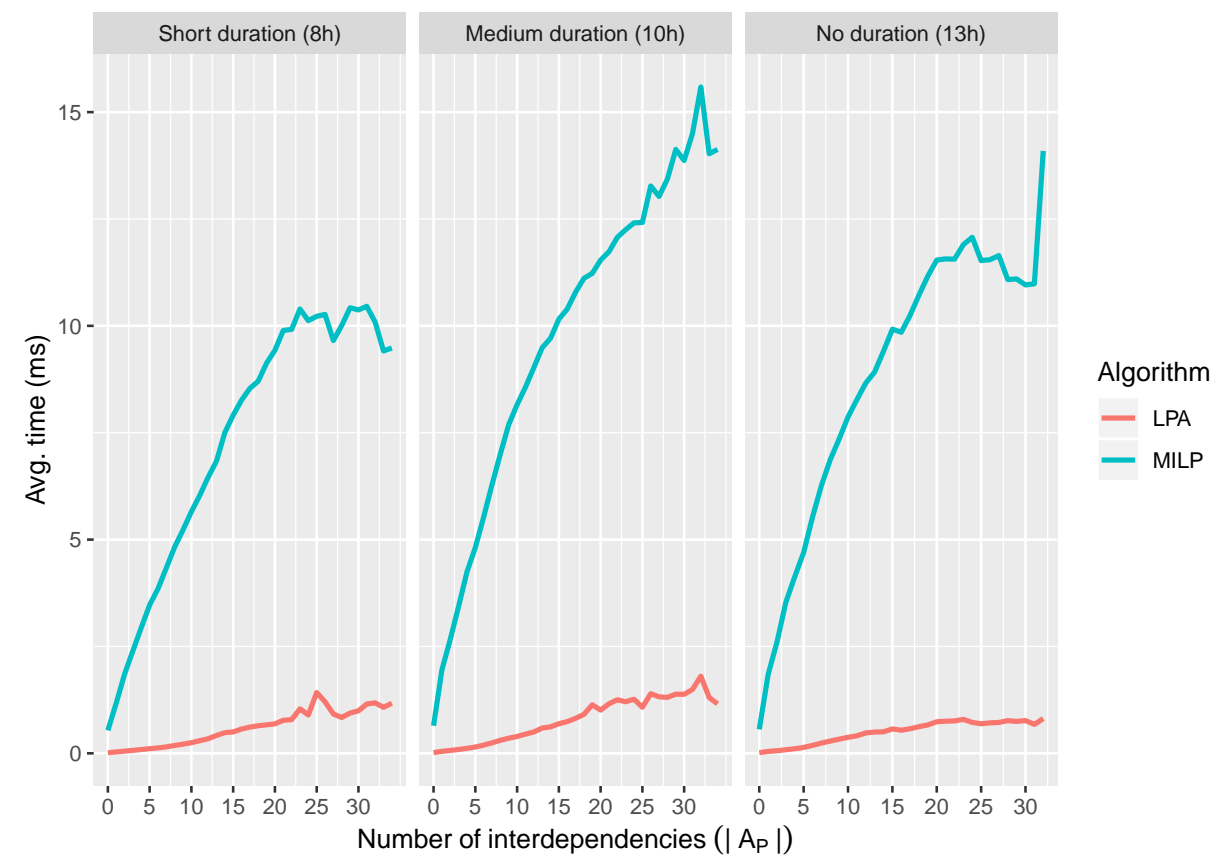

In terms of the total computation time, the LPA solved all feasible TDSP-IR instances in 51 minutes, while the MILP required 934 minutes. 
Therefore, the LPA remains almost 20 times faster than the MILP.

The computational effort required by the LPA depends on the number of propagated labels. Meanwhile, the number of labels that need to be propagated to prove infeasibility depends not only on the size of the instance, but also on the topological order. If two nodes which create a conflict are at the beginning of $\mathcal{T}_{G}$, then infeasibility is detected quickly. However, when these nodes are located towards the end of $\mathcal{T}_{G}$, the number of propagations can grow significantly, increasing the computational burden. Indeed, all instances which require long computation times of the LPA contain hundreds of nodes and $\left|A_{P}\right| \geq 18$ interdependencies. Thus, there is a large probability that conflicts will take many propagations to be detected due to the long topological order in these instances.

Note that identifying the conflicting nodes and deciding the best topological order in advance are nontrivial tasks and remain as an open question. Meanwhile, proving feasibility only requires finding one solution and can be achieved much faster by the LPA, as demonstrated by Figure 6 .

\subsection{Comparison to a naive scheduling method}

To understand both the importance of considering the interdependencies when scheduling breaks and the TDSP-IR instances' difficulty, we have implemented a naive algorithm inspired by Goel (2009). In contrast to the LPA, which is a multi-label algorithm, the naive approach uses only one label and schedules each route independently with breaks as late as possible. Once a schedule for each route has been generated, feasibility is verified using the STP described in Section 4.5 .

Table 3 shows the percentage of feasible solutions found by the naive method compared to both the LPA and the MILP (both of which find 100\% of the solutions). The accuracy ratio is based on all feasible instances for which $\left|A_{P}\right| \leq q$, for a given value $q \geq 0$. The single-route TDSP is encountered when $q=0$, while $q \geq 1$ refers strictly to TDSP-IR instances. Results are grouped according to their maximum duration $M_{\text {dur }}$ as this parameter has some influence over the degree of difficulty of the instances. Given that the execution times of the naive algorithm were always below $0.1 \mathrm{~ms}$, we do not report them in the table.

The ratios for the single-route case $\left(\left|A_{P}\right|=0\right)$ are similar to those reported by Goel (2010) for routes containing between 6 and 7 customers. The average number of locations in our instances was 12 , but the scheduling was also arguably simpler by considering only one working day. Therefore, the results for the single-route case are within the expected ratios, and even in this simple case the naive approach misses many feasible solutions.

Once instances with one interdependent task are considered $\left(\left|A_{P}\right|=1\right)$, the ratios of the naive method drop by as much as $4 \%$. Further increases in the size of $A_{P}$ always decrease the solution ratios of the naive approach. When accounting for the more complex and large instances $\left(\left|A_{P}\right| \leq 30\right)$, 
Table 3: Ratio (\%) of feasible solutions found by the scheduling algorithms.

\begin{tabular}{|c|c|c|c|c|}
\hline \multirow[b]{2}{*}{ Algorithm } & \multirow[b]{2}{*}{$\left|A_{P}\right|$} & \multicolumn{3}{|c|}{ Duration $M_{\text {dur }}$} \\
\hline & & $8 \mathrm{~h}$ & $10 \mathrm{~h}$ & $13 \mathrm{~h}$ \\
\hline LPA/MILP & all & 100.00 & 100.00 & 100.00 \\
\hline \multirow{7}{*}{ Naive } & $=0$ & 83.93 & 84.62 & 86.81 \\
\hline & $\leq 1$ & 82.49 & 80.16 & 82.07 \\
\hline & $\leq 2$ & 81.53 & 76.56 & 78.61 \\
\hline & $\leq 3$ & 80.82 & 74.05 & 75.56 \\
\hline & $\leq 4$ & 80.28 & 71.28 & 73.10 \\
\hline & $\leq 5$ & 79.80 & 68.95 & 70.46 \\
\hline & $\leq 30$ & 76.40 & 44.63 & 39.67 \\
\hline
\end{tabular}

the ratios drop to no more than $76 \%$, but reach as little as $39 \%$ of the feasible solutions found. All of these results consider the single-route case in their ratios, which makes the Naive method appear more accurate than it really is. Alternatively, when taking into account only the TDSP-IR instances (those where $1 \leq\left|A_{P}\right| \leq 30$ ), the accuracy ratio is only $19.17 \%$, $8.92 \%$ and $8.85 \%$ for durations of $8 \mathrm{~h}, 10 \mathrm{~h}$ and $13 \mathrm{~h}$, respectively. These results clearly demonstrate how problematic it is to ignore interdependencies when scheduling the drivers' HOS.

Additionally, for small sizes of $\left|A_{P}\right|$ it is easier for the naive algorithm to find solutions for longer time horizons due to routes which are less restricted when it comes to positioning breaks. However, once the size exceeds the $\left|A_{P}\right| \geq 4$ threshold, the naive algorithm finds fewer feasible solutions when the time horizon increases as this is also related to an increase in the number of nodes and routes requiring the careful scheduling of breaks.

Finally, the results in Table 3 demonstrate the importance of considering the interplay between interdependencies and the scheduling of breaks for truck drivers. If the algorithms described in this paper are to be used in VRP solvers, it is undesirable to employ feasibility tests that miss $20 \%$ of the feasible solutions $\left(\left|A_{P}\right|=0\right)$, let alone $25-60 \%$ of them. Therefore, it is worth considering that the LPA presented a good trade-off between execution time and feasibility ratio and can thus be used to quickly answer decision questions regarding TDSP-IR instances for real-world applications.

\section{Conclusion and future work}

Research concerning the Truck Driver Scheduling Problem had, until now, mainly focused on the scheduling of independent routes. This sets limitations for real-world applications since solution techniques for problems such as the Pickup and Delivery Problem with Transshipments could not effectively guarantee compliance with hours of service regulations due to the presence of interdependent routes. 
The research reported in this paper has successfully closed this gap by introducing a Mixed Integer Programming formulation and a Label Propagation Algorithm capable of scheduling interdependent routes while respecting a set of EU regulations for truck drivers. One major advantage of the proposed Label Propagation Algorithm is that it can be easily applied to any vehicle routing solver that addresses minimum difference interdependencies.

Despite being easy to incorporate the Label Propagation Algorithm within a vehicle routing solver, doing so can result in lengthy computational runtimes given that hundreds, or perhaps even thousands, of solutions must be evaluated per iteration. To overcome this potential overhead, variants of the scheduling algorithm could be considered for further research. For example, one could employ a bidirectional labeling algorithm, which was shown to be effective for the single-route case (Tilk \& Goel, 2020). Another option could be to maintain only a subset of labels after each propagation, thus solving the problem heuristically, similar to the approach by Goel \& Vidal (2013). An experimental study should be conducted, however, to ensure that a reasonable amount of feasible solutions is produced by the heuristic according to each specific application.

Furthermore, many interesting scientific challenges remain open. An obvious question relates to the theoretical complexity of the Truck Driver Scheduling Problem with Interdependent Routes. Is the problem solvable in polynomial time? Additionally, are there speedup techniques for the search or faster ways to prove an instance infeasible? Is it possible to minimize total route duration? Of course, one must first define what total route duration means for a set of interdependent vehicle routes: is it the sum of all durations? Is it the longest duration? Or is it the difference between the longest and shortest durations? Regardless of the definition, the computational runtimes are likely to increase significantly. Finally, what is the impact of including split breaks, rest periods and other EU-related regulations?

In terms of generalizing the algorithm to accommodate other regulations, this is clearly an interesting research direction of practical relevance because it would result in a single algorithm being capable of handling multiple sets of regulations. However, when addressing interdependent routes under certain regulations it is difficult to define effective label dominance criteria to speed up the label propagation algorithm. Nevertheless, an algorithm that accommodates multiple regulations would likely be more applicable if the regulations incorporated were from neighboring countries such as the United States and Canada (or Mexico) where many truck drivers cross the border on a daily basis to transport goods between the countries. This poses a scientific challenge which requires further investigation for both the singleand multiple-route truck driver scheduling problems.

Finally, from a broader perspective, one may consider the impact that hours of service regulations have on the use of transfer locations and crossdocks in logistic applications. It should be expected that within a longer time 
horizon, it is preferable for routes of limited duration to utilize transfers to partially serve more requests in order to make the most of otherwise useless trips. However, this remains an open research topic.

\section{Acknowledgments}

The authors are thankful for the valuable contributions of the two anonymous reviewers that improved the quality the paper. This research received financial support from the Strategic Basic Research project Data-driven logistics $[\mathrm{S} 007318 \mathrm{~N}]$ funded by the Research Foundation Flanders (FWO), and from the Flemish Government under the Artificiële Intelligentie (AI) Vlaanderen Programme. Editorial consultation provided by Luke Connolly (KU Leuven).

\section{References}

Cormen, T. H., Leiserson, C. E., Rivest, R. L., \& Stein, C. (2009). Introduction to Algorithms. MIT press.

Dechter, R., Meiri, I., \& Pearl, J. (1991). Temporal constraint networks. Artificial Intelligence, 49, $61-95$.

Dohn, A., Rasmussen, M. S., \& Larsen, J. (2011). The vehicle routing problem with time windows and temporal dependencies. Networks, 58, 273-289.

Drexl, M. (2013). Applications of the vehicle routing problem with trailers and transshipments. European Journal of Operational Research, 227, 275 -283 .

European Commission (2002). Directive 2002/15/EC on the organisation of the working time of persons performing mobile road transport activities. Official J. Eur. Commun. L 80 3539. Available online at: http://data. europa.eu/eli/dir/2002/15/oj (last access: 20-04-2021).

European Commission (2006). Regulation (EC) no. 561/2006 on the harmonisation of certain social legislation relating to road transport and amending council regulations (EEC) no 3821/85 and (EC) no. 2135/98 and repealing council regulation (EEC) no. 3820/85. Official J. Eur. Union L 102, 11.04.2006. Available online at: http://data.europa.eu/eli/ reg/2006/561/oj (last access: 20-04-2021).

Goel, A. (2009). Vehicle scheduling and routing with drivers' working hours. Transportation Science, 43, 17-26.

Goel, A. (2010). Truck driver scheduling in the European Union. Transportation Science, 44, 429-441. 
Goel, A. (2012). The minimum duration truck driver scheduling problem. EURO Journal on Transportation and Logistics, 1, 285-306.

Goel, A. (2014). Hours of service regulations in the United States and the 2013 rule change. Transport Policy, 33, $48-55$.

Goel, A. (2018). Legal aspects in road transport optimization in Europe. Transportation Research Part E: Logistics and Transportation Review, $114,144-162$.

Goel, A., Archetti, C., \& Savelsbergh, M. (2012). Truck driver scheduling in Australia. Computers \& Operations Research, 39, 1122 - 1132.

Goel, A., \& Irnich, S. (2017). An exact method for vehicle routing and truck driver scheduling problems. Transportation Science, 51, 737-754.

Goel, A., \& Kok, L. (2012). Truck driver scheduling in the United States. Transportation Science, 46, 317-326.

Goel, A., \& Rousseau, L.-M. (2012). Truck driver scheduling in Canada. Journal of Scheduling, 15, 783-799.

Goel, A., \& Vidal, T. (2013). Hours of service regulations in road freight transport: An optimization-based international assessment. Transportation Science, 48, 391-412.

Kleff, A. (2019). Scheduling and Routing of Truck Drivers Considering Regulations on Drivers' Working Hours. Ph.D. thesis Karlsruher Institut für Technologie (KIT). doi:10.5445/IR/1000097855.

Kok, A., Hans, E., \& Schutten, J. (2011). Optimizing departure times in vehicle routes. European Journal of Operational Research, 210, 579 - 587.

Leung, J. Y. (2004). Handbook of scheduling: algorithms, models, and performance analysis. CRC press.

Masson, R., Lehuédé, F., \& Péton, O. (2013). Efficient feasibility testing for request insertion in the pickup and delivery problem with transfers. Operations Research Letters, 41, $211-215$.

Masson, R., Lehuédé, F., \& Péton, O. (2014). The dial-a-ride problem with transfers. Computers \& Operations Research, 41, $12-23$.

Mitrović-Minić, S., \& Laporte, G. (2006). The pickup and delivery problem with time windows and transshipment. INFOR: Information Systems and Operational Research, 44, 217-227.

Sampaio, A., Savelsbergh, M., Veelenturf, L. P., \& Van Woensel, T. (2020). Delivery systems with crowd-sourced drivers: A pickup and delivery problem with transfers. Networks, 76, 232-255. 
Tilk, C., \& Goel, A. (2020). Bidirectional labeling for solving vehicle routing and truck driver scheduling problems. European Journal of Operational Research, 283, 108-124.

Van Belle, J., Valckenaers, P., \& Cattrysse, D. (2012). Cross-docking: State of the art. Omega, 40, 827-846. 


\section{Appendices}

\section{A. Dedicated topological order}

This appendix describes the topological sorting algorithm applied in the Toposort function from Algorithm 1. The idea is to benefit from the structure of the problem to achieve shorter execution times for the LPA, while having no proven impact on the theoretical worst-case complexity of the algorithm.

\section{A.1. Impact of topological order}

To understand the impact of a specific topological order, consider the instance with $m=2$ routes depicted in Figure 7. Assume that $M_{\text {drive }}=7$, $B=1$ and consider $\mathcal{T}_{G}^{1}=\left(a, b_{-}, c, e, b_{+}, f, d_{2}, d_{1}\right)$. For simplicity, route duration and working times are ignored.

Algorithm 1 starts with initial label $\mathcal{L}^{0}$ and calls Algorithm 2 which in turn uses the LabelEnumeration function. This function follows $\mathcal{T}_{G}^{1}$ and propagates labels in route $r_{1}$ until node $c$ is reached. The next propagation (to $e$ ) refers to route $r_{2}$, in which case Algorithm 2 terminates and returns to Algorithm 1 for the next iteration. Thus, upon scheduling $c$ there are three non-dominated labels in the expansion $-\mathcal{L}^{1}, \mathcal{L}^{2}$ and $\mathcal{L}^{3}$ - which have no break scheduled, a break after $a$, and a break after $b_{-}$, respectively. Table 4 reports the values of these labels. Note that the expansion up to $c$ generated four labels, but the label with two breaks (after $a$ and $b_{-}$) is dominated by $\mathcal{L}^{3}$ (and thus removed from the search).

As for the other dominance tests, label $\mathcal{L}^{1}$ cannot dominate any other label because it requires more driving time. Label $\mathcal{L}^{2}$ has less available slack and push than the others, but visits $c$ earlier than $\mathcal{L}^{3}$. Finally, label $\mathcal{L}^{3}$ visits $c$ later than any other label, but with the least driving time.

Continuing the propagation, each of the three labels previously expanded up to $c$ in route $r_{1}$ are now expanded from $\mathcal{L}^{1}\left(i_{2}\right)=\mathcal{L}^{2}\left(i_{2}\right)=\mathcal{L}^{3}\left(i_{2}\right)=o_{2}$ until the destination node $d_{2}$. The propagation generates three non-dominated labels (break at $e$, break at $b_{+}$, and break at both) for each one of the three labels up to $d_{1}$ (enumerating the valid combination of breaks in every route). Note that no break at all is infeasible. Thus, the total number of labels upon reaching $d_{2}$ is nine.

Now consider a different topological order, such that $d_{1}$ is reached before the propagation in $r_{2}$ begins, that is, $\mathcal{T}_{G}^{2}=\left(a, b_{-}, c, d_{1}, e, b_{+}, f, d_{2}\right)$. The same three non-dominated labels are generated up to node $c$. Expanding them up to $d_{1}$ generates a total of six new labels (for each of the three labels up to $c$, it either breaks after $c$ or has a direct trip to $d_{1}$ ). However, expanding $\mathcal{L}^{1}$ and $\mathcal{L}^{2}$ without a break after $c$ are both infeasible, thus only four feasible and consistent labels remain. Let us denote these labels $\mathcal{L}^{4}$ 
(break at $c), \mathcal{L}^{5}$ (break at $\left.b_{-}\right), \mathcal{L}^{6}$ (break at $a$ and $c$ ), and $\mathcal{L}^{7}$ (break at $b_{-}$ and $c$ ). Table 5 reports their values.

Figure 7: Instance to demonstrate the differences incurred by topological order.

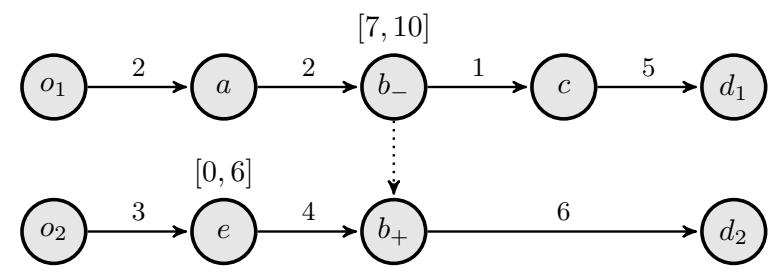

Table 4: Three labels in the expansion with $\mathcal{T}_{G}^{1}$ up to $c$.

\begin{tabular}{|c|ccccccc|}
\hline Lab. & $p$ & $i_{1}$ & $i_{2}$ & $H_{c}$ & $D_{c}$ & $P_{1}$ & $S_{1 c}$ \\
\hline $\mathcal{L}^{1}$ & 4 & $c$ & $o_{2}$ & 8 & 5 & 6 & 3 \\
$\mathcal{L}^{2}$ & 4 & $c$ & $o_{2}$ & 8 & 3 & 5 & 2 \\
$\mathcal{L}^{3}$ & 4 & $c$ & $o_{2}$ & 9 & 1 & 6 & 3 \\
\hline
\end{tabular}

Table 5: Four labels in the expansion with $\mathcal{T}_{G}^{2}$ up to $d_{1}$.

\begin{tabular}{|c|ccccccc|}
\hline Lab. & $p$ & $i_{1}$ & $i_{2}$ & $H_{d_{1}}$ & $D_{d_{1}}$ & $P_{1}$ & $S_{1 d_{1}}$ \\
\hline $\mathcal{L}^{4}$ & 5 & $d_{1}$ & $o_{2}$ & 14 & 1 & 6 & 3 \\
$\mathcal{L}^{5}$ & 5 & $d_{1}$ & $o_{2}$ & 14 & 6 & 6 & 3 \\
$\mathcal{L}^{6}$ & 5 & $d_{1}$ & $o_{2}$ & 14 & 5 & 5 & 2 \\
$\mathcal{L}^{7}$ & 5 & $d_{1}$ & $o_{2}$ & 15 & 5 & 6 & 3 \\
\hline
\end{tabular}

Looking closely at dominance tests, we note that label $\mathcal{L}^{4}$ dominates $\mathcal{L}^{5}$, $\mathcal{L}^{6}$ and $\mathcal{L}^{7}$. Therefore, when the propagation from $o_{2}$ until $d_{2}$ begins for route $r_{2}$, only one non-dominated label remains $\left(\mathcal{L}^{4}\right)$. As with the first topological order, upon reaching $d_{2}$ three non-dominated schedules were generated per label that reached, in this case, $d_{1}$. Since there was only one label at $d_{1}$, the total number of labels upon reaching $d_{2}$ is three. The reduction is therefore two-thirds when compared to the first topological order.

\section{A.2. Procedure for the dedicated topological order}

To obtain a topological order $\mathcal{T}_{G}$, we propose the following procedure. Associate with each route $r_{k}$ a value $\delta_{k}$ that contains the total number of $u_{+}$nodes belonging to route $r_{k}$ (recall that by definition $u_{+}$belongs to a pair $\left.\left(u_{-}, u_{+}\right) \in A_{P}\right)$. Furthermore, let $\left|r_{k}\right|$ denote the number of locations in the $k$-th route and $\rho_{k}$ a penalty value, which is initialized with a value of zero for all routes.

Our procedure begins by sorting routes in ascending order of $\delta_{k}$. Ties are broken by descending order of $\left|r_{k}\right|$. Then, the procedure takes the first route after sorting, denoted $r_{z}$ (the route with the smallest $\delta_{z}$ ), and adds as many nodes as possible from this route to the topological order $\mathcal{T}_{G}$. Nodes 
from $r_{z}$ are no longer included either when $d_{z}$ is added to the order (the route ends) or when a node $u_{+}$is reached for which the corresponding $u_{-}$ has not been added to the order. In the latter case, $\rho_{z}$ is increased by one unit. Whenever a $u_{-}$node is included in $\mathcal{T}_{G}$, take the route $r_{y}$ of the corresponding $u_{+}$node and decrease the values $\delta_{y}$ and $\rho_{y}$ by one unit. Once nodes of a route cannot be added to the order, re-sort routes in ascending order of $\rho_{k}$, then ascending order of $\delta_{k}$, and finally descending order of $\left|r_{k}\right|$. When a destination $d_{k}$ is added to $\mathcal{T}_{G}$, route $r_{k}$ is no longer considered.

In this way, whenever a route $r_{z}$ has no unresolved dependencies $\left(\delta_{z}=0\right)$ it is preferred for inclusion in the topological order. By adding as many nodes of only one route as possible, many routes will be completed one after the other, thereby avoiding back-and-forth propagations between different routes. Meanwhile, penalties $\rho$ are used to avoid cases in which a mutual dependency could cause an infinite loop in the sorting function, such as in Figure 8 ,

This example shows the reasoning behind penalty $\rho_{k}$ when using the specific Toposort described in the paper. A mutual dependency is depicted in Figure 8, where $\delta_{1}=1$ and $\delta_{2}=1$. Sorting these routes has two possible outcomes. The first case begins with $r_{1}$, which can add nodes to the order up to $a_{-}$(inclusive), reducing $\delta_{2}=0$. Re-sorting the routes makes $r_{2}$ the next to be processed. Finally, adding all nodes in $r_{2}$ and then completing $r_{1}$ ends the topological order. Alternatively, if $r_{2}$ is the first route, the procedure can only add $\mathrm{O}_{2}$ before reaching an unresolved dependency. Re-sorting routes without penalty $\rho_{k}$ does not change anything since no $\delta_{k}$ value was modified. If a penalty is included in the sorting and increased once $a_{+}$is reached, route $r_{1}$ will be preferred after the re-sorting step.

Figure 8: An instance depicting mutual dependencies.

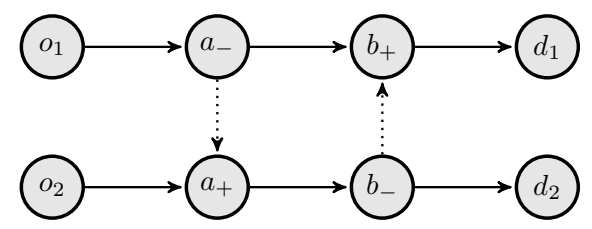

The proposed sorting is clearly more complex than a simple topological sorting. However, its computational cost is compensated for by the observed performance gains. In terms of runtime complexity, the initial sorting of routes takes $\mathcal{O}(m \log m)$ with a standard sorting algorithm. In the worst case, the algorithm may have to re-sort routes after each node insertion, thus leading to an overall complexity of $\mathcal{O}(n m \log m)$.

\section{B. Detailed examples}

Sections B.1 and B.2 present examples to help justify label dominance Conditions (33) and (34). The variables used are those listed in Table 2 
unless otherwise stated.

\section{B.1. Example for dominance Condition (33)}

To help clarify Condition (33), consider Figure 9. Suppose $M_{\text {drive }}=15$, $M_{\text {dur }}=30, B=1$, and $\mathcal{T}_{G}=\left(a, b, c_{-}, e, d_{1}, f, c_{+}, d_{2}\right)$. A break is required in $r_{1}$ at some point before $c_{-}$. When scheduling $r_{1}$, the algorithm reaches node $e$ with two labels $\mathcal{L}^{1}$ and $\mathcal{L}^{2}$. For $\mathcal{L}^{1}\left(x_{a}\right)=1$ (break after $a$ ), whereas for $\mathcal{L}^{2}\left(x_{b}\right)=1$ (break after $b$ ). The variables for both labels when $\mathcal{L}^{1}\left(i_{1}\right)=$ $\mathcal{L}^{2}\left(i_{1}\right)=e$ are detailed in Table 6 .

Figure 9: A TDSP-IR instance highlighting the necessity for Condition 33.

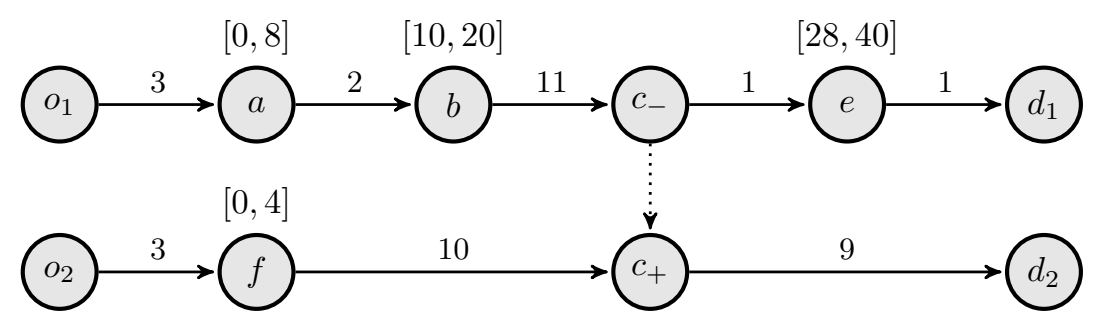

Table 6: Two non-dominated labels at node $e$ for the example in Figure 9

\begin{tabular}{|c|ccccccc|}
\hline Lab. & $p$ & $i_{1}$ & $i_{2}$ & $H_{e}$ & $D_{e}$ & $P_{1}$ & $S_{1 e}$ \\
\hline $\mathcal{L}^{1}$ & 4 & $e$ & $o_{2}$ & 28 & 14 & 5 & 10 \\
$\mathcal{L}^{2}$ & 4 & $e$ & $o_{2}$ & 28 & 12 & 5 & 10 \\
\hline
\end{tabular}

All variables are identical, except for $D_{e}$. A break after $b$ appears to be the best schedule since it serves $e$ at the same time for both $\mathcal{L}^{1}$ and $\mathcal{L}^{2}$ and keeps the same $P_{1}$ and $S_{1 e}$ values, but with less driving time. Without further investigation, one could simply say that if $\mathcal{L}^{1}$ is feasible, then $\mathcal{L}^{2}$ must also be feasible, and it is therefore only necessary to propagate $\mathcal{L}^{2}$.

However, let us consider what happens when propagation continues until the end of $\mathcal{T}_{G}$. Normally, the propagation from $e$ to $d_{1}$ would generate two labels for each label upon $e$, making for a total of 4 labels. Since it is unnecessary to schedule a break after $e$, we ignore the labels for which $x_{e}=1$ and consider only labels $\mathcal{L}^{1}$ and $\mathcal{L}^{2}$ with a direct trip from $e$ to $d_{1}$. After that, further propagations continue in $r_{2}$.

A break is required after $c_{+}$to comply with driving regulations $M_{\text {drive }}$. Considering label $\mathcal{L}^{1}$, route $r_{2}$ will have a waiting time of 7 at $c_{+}$because $\mathcal{L}^{1}\left(H_{c_{-}}\right)=21$. Meanwhile, for $\mathcal{L}^{2}$ there is a waiting time of 8 units because $\mathcal{L}^{2}\left(H_{c_{-}}\right)=22$. Note that including the break after $c_{+}$as required, that is setting $\mathcal{L}^{1}\left(x_{c_{+}}\right)=\mathcal{L}^{2}\left(x_{c_{+}}\right)=1$ changes the arrival times $\mathcal{L}^{1}\left(H_{d_{2}}\right)=31$ and $\mathcal{L}^{2}\left(H_{d_{2}}\right)=32$. Due to node $f$, the driver in route $r_{2}$ cannot depart from $o_{2}$ later than at time $H_{o_{1}}=1$ and therefore the duration of route $r_{2}$ in label $\mathcal{L}^{1}$ 
is feasible, while in $\mathcal{L}^{2}$ it is not. In other words, while $\operatorname{STP}\left(\mathcal{L}^{1}\right)$ is consistent, $\operatorname{STP}\left(\mathcal{L}^{2}\right)$ has a negative cycle and is thus inconsistent.

The problem in this example is that $\mathcal{L}^{1}\left(H_{e}\right)=\mathcal{L}^{2}\left(H_{e}\right)$. In other words, the two drivers begin service at node $e$ at the same time, due to the waiting time at $e$. The only difference between variables in $\mathcal{L}^{1}$ and $\mathcal{L}^{2}$ is the start time of service at node $c_{-}$. However, upon scheduling $e$, the information from $c_{-}$would not be considered anymore in dominance tests unless we include Condition (33).

\section{B.2. Example for dominance Condition (34)}

By way of example, consider Figure 10. Suppose $M_{\text {drive }}=12, M_{\text {dur }}=15$, $B=1$ and $\mathcal{T}_{G}=\left(a_{-}, d_{1}, b, a_{+}, c_{-}, e_{-}, d_{2}, c_{+}, e_{+}, f, d_{3}\right)$. It is self-evident that no break is required in route $r_{1}$, while a break is required in $r_{2}$ at either $b, a_{+}, c_{-}$or $e_{-}$. Let $\mathcal{L}^{1}$ be the label with a break scheduled after $b, \mathcal{L}^{2}$ the label with a break at $a_{+}, \mathcal{L}^{3}$ the label with a break at $c_{-}$, and $\mathcal{L}^{4}$ the label with a break at $e_{-}$. Consider their expansion up to $d_{2}$. Table 7 reports the values for each label.

Figure 10: A TDSP-IR instance showing the need for Condition 34 .

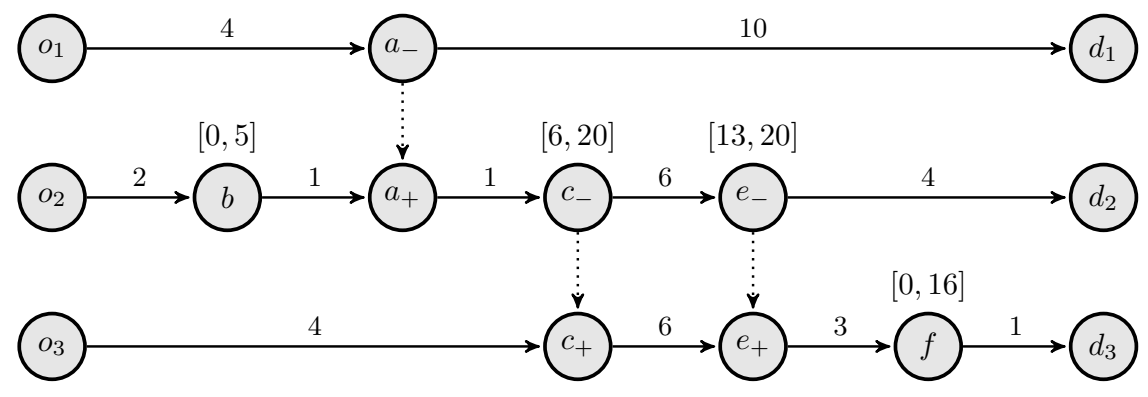

Table 7: Labels active upon reaching node $d_{2}$.

\begin{tabular}{|c|ccccccccccc|}
\hline Lab. & $p$ & $i_{1}$ & $i_{2}$ & $i_{3}$ & $H_{d_{2}}$ & $D_{d_{2}}$ & $P_{1}$ & $P_{2}$ & $S_{2 c_{-}}$ & $S_{2 d_{2}}$ & $S_{1 d_{2}}$ \\
\hline $\mathcal{L}^{1}$ & 7 & $d_{1}$ & $d_{2}$ & $o_{3}$ & 17 & 12 & 9 & 3 & 1 & 2 & 2 \\
$\mathcal{L}^{2}$ & 7 & $d_{1}$ & $d_{2}$ & $o_{3}$ & 17 & 11 & 8 & 3 & 1 & 2 & 1 \\
$\mathcal{L}^{3}$ & 7 & $d_{1}$ & $d_{2}$ & $o_{3}$ & 17 & 10 & 8 & 3 & 2 & 2 & 1 \\
$\mathcal{L}^{4}$ & 7 & $d_{1}$ & $d_{2}$ & $o_{3}$ & 18 & 4 & 9 & 3 & 2 & 3 & 2 \\
\hline
\end{tabular}

At first, the only valid dominance is $\mathcal{L}^{3}$ over $\mathcal{L}^{2}$, because $\mathcal{L}^{3}$ requires less driving time up to $d_{2}$ while the remaining values are the same as for $\mathcal{L}^{2}$. Thus, $\mathcal{L}^{2}$ is no longer considered. As for the other labels, $\mathcal{L}^{3}$ does not dominate $\mathcal{L}^{1}$ because it has less slack $S_{1 d_{2}}$. Label $\mathcal{L}^{4}$ cannot dominate the other two labels because it arrives later at $d_{2}$.

Due to time windows, all labels serve requests $c_{-}$and $e_{-}$at the same time: $\mathcal{L}^{1}\left(H_{c_{-}}\right)=\mathcal{L}^{3}\left(H_{c_{-}}\right)=\mathcal{L}^{4}\left(H_{c_{-}}\right)=6$ and $\mathcal{L}\left(H_{e_{-}}\right)^{1}=\mathcal{L}\left(H_{e_{-}}\right)^{3}=$ 
$\mathcal{L}\left(H_{e_{-}}\right)^{4}=13$. However, if we ignore values $D_{d_{2}}$ (because route $r_{2}$ has been concluded), there are two new dominances: $\mathcal{L}^{1}$ over $\mathcal{L}^{3}$ and $\mathcal{L}^{1}$ over $\mathcal{L}^{4}$. Note that $\mathcal{L}^{1}$ over $\mathcal{L}^{3}$ is only valid if Condition 34 is not included in the dominance test. Moreover, label $\mathcal{L}^{4}$ was only removed by ignoring variable $D_{d_{2}}$, which would otherwise be propagated unnecessarily until the end.

Consider the expansion of $r_{3}$, which can only have a break scheduled after $c_{+}$(a break after $e_{+}$is infeasible due to the time window at $f$ ). After expanding label $\mathcal{L}^{1}$ (the only one remaining), we discover that $\operatorname{STP}\left(\mathcal{L}^{1}\right)$ is not consistent, because route $r_{2}$ needs to be postponed such that service at $\mathcal{L}^{1}\left(H_{c_{-}}\right)=7$. However, this would require service at $\mathcal{L}^{1}\left(H_{f}\right)=16>l_{f}$. Therefore, the conclusion would be that this instance is infeasible.

If we include Condition (34), label $\mathcal{L}^{3}$ is not removed. Indeed, following the same steps as before shows that $\mathcal{L}^{3}$ is consistent because route $r_{2}$ is feasible with $\mathcal{L}^{3}\left(H_{c_{-}}\right)=6$.

\section{Proof of Lemma 1}

This Appendix presents the detailed proof that the label dominance Conditions 28 - (34) only remove labels for which another label exists that can produce a feasible schedule, should one exist. The variables used are those in the main body of the paper, unless otherwise stated. For simplicity, we denote the latest scheduled node in any route $r_{k}\left(\mathcal{L}\left(i_{k}\right)=\mathcal{L}^{\prime}\left(i_{k}\right)\right)$ as $i_{k}$.

Proof. Let us define two labels $\mathcal{L}$ and $\mathcal{L}^{\prime}$. As an initial hypothesis, suppose $\mathcal{L}$ dominates $\mathcal{L}^{\prime}$. Then, we must show that any future modification in $\mathcal{L}^{\prime}$ will impact all the $m$ schedules at least as much as $\mathcal{L}$.

Consider any route index $k=1, \ldots, m$. Conditions 28)-(31) assure that: $\mathcal{L}\left(H_{i_{k}}\right) \leq \mathcal{L}^{\prime}\left(H_{i_{k}}\right), \mathcal{L}\left(D_{i_{k}}\right) \leq \mathcal{L}^{\prime}\left(D_{i_{k}}\right), \mathcal{L}\left(W_{i_{k}}\right) \leq \mathcal{L}^{\prime}\left(W_{i_{k}}\right)$, and $\mathcal{L}\left(P_{k}\right) \geq \mathcal{L}^{\prime}\left(P_{k}\right)$. Suppose $\mathcal{L}\left(H_{i_{k}}\right)=\mathcal{L}^{\prime}\left(H_{i_{k}}\right)$. If $\mathcal{L}^{\prime}$ can create a feasible schedule for $r_{k}$, then $\mathcal{L}$ must also create a feasible schedule for this route since it requires fewer resources $\left(D_{i_{k}}\right.$ and $\left.W_{i_{k}}\right)$ to reach the same location $\left(i_{k}\right)$ at the same time $\left(H_{i_{k}}\right)$, while having more opportunities to postpone the start time $\left(P_{k}\right)$. This is also true when $\mathcal{L}\left(H_{i_{k}}\right)<\mathcal{L}^{\prime}\left(H_{i_{k}}\right)$ since it is possible to purposefully postpone the service at $i_{k}$ in label $\mathcal{L}$ such that $\mathcal{L}\left(H_{i_{k}}\right)=\mathcal{L}^{\prime}\left(H_{i_{k}}\right)$.

For Condition (32), we verify that all slack from origins $o_{z}, z \in \Phi\left(i_{k}\right)$ up to $i_{k}$ are at least as large for $\mathcal{L}$ as they are for $\mathcal{L}^{\prime}$ to ensure compliance with maximum route duration. This guarantees that any postponement to the start time of a route in $\mathcal{L}$ will impact other routes at least as much as the same postponement in $\mathcal{L}^{\prime}$.

Suppose the start time of service at origin $o_{z}$ has to be shifted forward in time by $\alpha_{z}$. In other words, the start times at $o_{z}, \mathcal{L}\left(H_{o_{z}}\right)$ and $\mathcal{L}^{\prime}\left(H_{o_{z}}\right)$ will be modified. This shift causes the following variable updates:

$$
\mathcal{L}\left(H_{o_{z}}\right) \leftarrow \mathcal{L}\left(H_{o_{z}}\right)+\alpha_{z}
$$




$$
\mathcal{L}^{\prime}\left(H_{o_{z}}\right) \leftarrow \mathcal{L}^{\prime}\left(H_{o_{z}}\right)+\alpha_{z}
$$

which implies $\mathcal{L}\left(H_{i_{k}}\right)$ in route $r_{k}$ will be shifted because origin $o_{z}$ precedes node $i_{k}\left(z \in \Phi\left(i_{k}\right)\right)$. The incurred shift is given by:

$$
\begin{aligned}
\mathcal{L}\left(H_{i_{k}}\right) & \leftarrow \mathcal{L}\left(H_{i_{k}}\right)+\max \left\{0, \alpha_{z}-\min \left\{\mathcal{L}\left(P_{z}\right), \mathcal{L}\left(S_{z i_{k}}\right)\right\}\right\} \\
\mathcal{L}^{\prime}\left(H_{i_{k}}\right) & \leftarrow \mathcal{L}^{\prime}\left(H_{i_{k}}\right)+\max \left\{0, \alpha_{z}-\min \left\{\mathcal{L}^{\prime}\left(P_{z}\right), \mathcal{L}^{\prime}\left(S_{z i_{k}}\right)\right\}\right\}
\end{aligned}
$$

Define $\mu_{i_{k}}=\max \left\{0, \alpha_{z}-\min \left\{\mathcal{L}\left(P_{z}\right), \mathcal{L}\left(S_{z i_{k}}\right)\right\}\right\}$ as the shift in $\mathcal{L}\left(H_{i_{k}}\right)$ (analogously for $\mathcal{L}^{\prime}$ we define $\mu_{i_{k}}^{\prime}$ ). Then, if $\mu_{i_{k}} \leq \mu_{i_{k}}^{\prime}$, it follows that $\min \left\{\mathcal{L}\left(P_{z}\right), \mathcal{L}^{\prime}\left(S_{z i_{k}}\right)\right\} \geq \min \left\{\mathcal{L}^{\prime}\left(P_{z}\right), \mathcal{L}\left(S_{z i_{k}}\right)\right\}$. From the initial hypothesis that $\mathcal{L}$ dominates $\mathcal{L}^{\prime}$, it is true that $\mathcal{L}\left(P_{k}\right) \geq \mathcal{L}^{\prime}\left(P_{k}\right)$ and that $\mathcal{L}\left(S_{z i_{k}}\right) \geq \mathcal{L}^{\prime}\left(S_{z i_{k}}\right)$, thus the inequality $\min \left\{\mathcal{L}\left(P_{z}\right), \mathcal{L}\left(S_{z i_{k}}\right)\right\} \geq \min \left\{\mathcal{L}^{\prime}\left(P_{z}\right), \mathcal{L}^{\prime}\left(S_{z i_{k}}\right)\right\}$ holds. Since $\alpha_{z}$ is a constant value, the resulting shift in $i_{k}$ must respect the relation $\mathcal{L}\left(H_{i_{k}}\right)+\mu_{i_{k}} \leq \mathcal{L}^{\prime}\left(H_{i_{k}}\right)+\mu_{i_{k}}^{\prime}$. Alternatively, $\mu_{i_{k}}>\mu_{i_{k}}^{\prime}$ would imply that $\min \left\{\mathcal{L}\left(P_{z}\right), \mathcal{L}\left(S_{z i_{k}}\right)\right\}>\min \left\{\mathcal{L}^{\prime}\left(P_{z}\right), \mathcal{L}^{\prime}\left(S_{z i_{k}}\right)\right\}$. Thus, either Condition (31) or Condition 32 fails and no dominance can be established, contradicting our initial hypothesis.

Condition (33) similarly checks the impact that a postponement on a service $u_{-}$in route $r_{k}$ may have on route $r_{z}\left(r_{k} \neq r_{z}\right)$ which has not been scheduled up to the corresponding $u_{+}$(for $\left(u_{-}, u_{+}\right) \in A_{P}$ ). In other words, there is no information concerning the slack, push, or service times at $u_{+}$. Since service time $\mathcal{L}\left(H_{u_{+}}\right)$may be defined by either its direct predecessors or by $\mathcal{L}\left(H_{u_{-}}\right)$, two distinct scenarios arise when analyzing this condition.

First, suppose that upon evaluating node $u_{+}$the algorithm finds the arrival time at $u_{+}$, here denoted by $E_{u_{+}}$, to be $E_{u_{+}}<\mathcal{L}\left(H_{u_{-}}\right)$. This means that there is a waiting time at $u_{+}$, which is computed by $\left(\mathcal{L}\left(H_{u_{-}}\right)+w_{u_{-}}\right)-E_{u_{+}}$. Clearly, for the same value $E_{u_{+}}$, the amount of waiting time increases proportional to $\mathcal{L}\left(H_{u_{-}}\right)$. Thus, unnecessary waiting time in route $r_{z}$ is minimized whenever $\mathcal{L}\left(H_{u_{-}}\right)$is minimized. By contrast, suppose $E_{u_{+}}>\mathcal{L}\left(H_{u_{-}}\right)$. In this case, no waiting occurs at node $u_{+}$and the proof focuses on the slack from origin $o_{k}$ to node $u_{+}$in route $r_{z}$. The increase in slack may include the term $\mathcal{L}\left(H_{u_{+}}\right)-\left(\mathcal{L}\left(H_{u_{-}}\right)+w_{u_{-}}\right)$(Equation 18), which is maximized whenever $\mathcal{L}\left(H_{u_{-}}\right)$is minimum for the same value $\mathcal{L}\left(H_{u_{+}}\right)$. However, this is not yet known when verifying Condition (33).

Hence, to guarantee minimum mandatory waiting time or maximum slack (both which contribute to ensuring maximum route duration), labels with minimum service time at $u_{-} \in U_{-}(\mathcal{L})$ nodes must be propagated until the corresponding $u_{+}$node is scheduled to generate complete information. Otherwise, a dominance could fail as per the example illustrated in Appendix B.1.

Condition (34) complements Condition (33) with the basic idea that if the labels have everything else equal, then it is necessary to look at the slack up to any given interdependent task $u_{-}$for which we do not know 
the start time of service at $u_{+}$. Essentially, for any origin $o_{z}$ in routes $r_{z}$ that precede $u_{-}$(that is, index $z \in \Phi\left(u_{-}\right)$), the impact of postponing $H_{o_{z}}$ on the start time of service at $u_{-}$should be minimal in order to establish dominance. Thus, if service in $o_{z}, H_{o_{z}}$ is postponed by $\alpha_{z}$, we have the following expressions:

$$
\begin{aligned}
\mathcal{L}\left(H_{u_{-}}\right) & \leftarrow \mathcal{L}\left(H_{u_{-}}\right)+\max \left\{0, \alpha_{z}-\min \left\{\mathcal{L}\left(P_{z}\right), \mathcal{L}\left(S_{z u_{-}}\right\}\right\}\right. \\
\mathcal{L}^{\prime}\left(H_{u_{-}}\right) & \leftarrow \mathcal{L}^{\prime}\left(H_{u_{-}}\right)+\max \left\{0, \alpha_{z}-\min \left\{\mathcal{L}^{\prime}\left(P_{z}\right), \mathcal{L}^{\prime}\left(S_{z u_{-}}\right\}\right\}\right.
\end{aligned}
$$

The proof continues in a similar way to what has been done for the general slack rule in Condition (32), except that in this case we look at slack up to all $u_{-} \in U_{-}(\mathcal{L})$ nodes, instead of looking at the latest scheduled node in each route. This clearly leads to a valid requirement that whenever Condition (34) is respected then, all else being equal, any postponement in routes preceding nodes $u_{-}$will impact the respective $u_{+}$service in $\mathcal{L}$ no more than in $\mathcal{L}^{\prime}$.

In conclusion, whenever Conditions (28)-(34) are satisfied for labels $\mathcal{L}$ and $\mathcal{L}^{\prime}$, either $\mathcal{L}$ produces a feasible schedule or neither $\mathcal{L}$ nor $\mathcal{L}^{\prime}$ can produce a feasible solution. Therefore, if $\mathcal{L}$ dominates $\mathcal{L}^{\prime}$, then $\mathcal{L}^{\prime}$ can be removed from the set of labels without incurring any loss of feasible solutions.

\section{Addressing United States regulations}

In order to solve the US-TDSP-IR, we must make a number of changes to the LPA. First, under US regulations for a single-working day, truck drivers must take a break of at least 30 minutes before no more than 8 hours have elapsed since the end of their last break or rest period (Goel, 2014). In our case, the last rest period refers to the beginning of the route. Note that ensuring no more than 8 hours of elapsed time differs significantly from the continuous driving time constraints present in the EU regulations. In the latter, the constraining time between two breaks is simply the total driving time, which is constant under our assumptions. In the US, however, the 8 hours of maximum elapsed time include driving and service times, which are constant, but also waiting times, which may increase depending on decisions taken in other routes due to the interdependencies. Furthermore, in the US, a break may have to be extended in duration so that the maximum elapsed time between two breaks is under $8 \mathrm{~h}$, whereas in the EU a break never needs to be longer than 45 minutes. 
To accommodate all of these conditions, we must first modify the STP formulation introduced in Section 4.5 which ensures feasibility of a label in the LPA. First, we associate with every node $i \in V$ in a TDSP-IR instance two variables $h_{i}^{\text {start }}$ and $h_{i}^{\text {end }}$, rather than a single variable as was the case for the EU regulations. Variable $h_{i}^{\text {start }}$ denotes the start time of service at node $i$ and $h_{i}^{\text {end }}$ denotes the departure time from node $i$. Then, given variable $h_{\alpha}=0$ which denotes the start of the time horizon, we can determine the feasibility of a label using $\operatorname{STP}(\mathcal{L})$ :

$$
\begin{aligned}
h_{i}^{\text {end }}-h_{j}^{\text {start }} \leq-t_{i j}, & \forall(i, j) \in A_{R} \\
h_{i}^{\text {start }}-h_{i}^{\text {end }} \leq-w_{i}-\mathcal{L}\left(x_{i}\right) \cdot B, & \forall i \in V \\
h_{u_{-}}^{\text {start }}-h_{u_{+}}^{\text {start }} \leq-w_{u_{-}}, & \forall\left(u_{-}, u_{+}\right) \in A_{P} \\
h_{\alpha}-h_{i}^{\text {start }} \leq-e_{i}, & \forall i \in V \\
h_{i}^{\text {start }}-h_{\alpha} \leq l_{i}, & \forall i \in V \\
h_{d_{k}}^{\text {end }}-h_{o_{k}}^{\text {start }} \leq M_{\text {dur }}, & \forall o_{k}, d_{k} \in V_{O}
\end{aligned}
$$

Whenever a new break is scheduled at a node $\beta$, an additional constraint must be enforced in the STP. Suppose that the last break in the route of node $\beta$ was scheduled at node $\lambda$. If no break was previously scheduled, $\lambda$ is simply the route's origin location. Then, the additional constraint is:

$$
h_{\beta}^{\text {start }}-h_{\lambda}^{\text {end }} \leq 8-w_{\beta}
$$

Given this formulation, we can obtain the precise length $B_{\beta}$ of the break at location $\beta$ by $B_{\beta}=h_{\beta}^{\text {end }}-h_{\beta}^{\text {start }}-w_{\beta}$.

However, the addition of this constraint effectively introduces a new arc in the STP-induced distance graph. This in turn increases the computational burden of solving the STP formulation with a shortest path algorithm. Moreover, the introduced formulation contains twice as many nodes as the formulation for the EU regulations.

The aforementioned changes only addressed the feasibility conditions of labels in the LPA. Let us now turn our attention to the challenges associated with the propagation and dominance criteria. When checking for the dominance of a label over another, we must ensure that service delays which may be required after further propagations have an impact that is not larger for one label than another. Delaying the start time of services may be required because either a break must be extended or the start time of previous services must be delayed. Either way, this would only occur in order to comply with the maximum elapsed time of $8 \mathrm{~h}$ between two breaks. Note that this is similar to ensuring maximum route duration in the EU. However, the key difference is that in the US any break may have to be postponed or extended, not just the departure time at the origin location of the route. Given that service delays caused by the postponement or extension 
of a break may affect multiple other routes by, for example, inducing waiting time at an interdependence location, it becomes non-trivial to ascertain dominance between two labels in an effective way. Could we somehow ensure that slack and push values for each break are greater for one label than another? But given that breaks are taken at different locations in different routes for each label, how should this comparison be made? How exactly can we define dominance between two labels in this situation? These are all interesting questions which open up exciting new lines of research for those who wish to study the TDSP-IR under US regulations.

In summary, it is not straightforward to define dominance between two labels. This implies that our best LPA implementation under US regulations is simply an exhaustive search algorithm and thus inefficient for any mediumsized instance.

\section{E. Supplementary results}

This appendix provides additional information for the computational results, including the specific modifications to standard PDPT instances in the literature, additional graphs and detailed tables.

\section{E.1. Modifications to Sampaio et al. (2020) instances}

The instances for the Pickup and Delivery Problem with Transshipment proposed by Sampaio et al. (2020) were modified in our experiments to better fit our experimental settings. The original instances considered a time horizon of no more than $\mathcal{H}=5 \mathrm{~h}$, which is short for our purposes. However, given that the instances were large (100-200 nodes) and readily available, we opted to modify them instead of creating new instances for the TDSP-IR experiments.

The modifications were restricted to: time horizon, time windows, and service times for customer locations. The time horizon of all instances was increased to $\mathcal{H}=13 \mathrm{~h}$ to fit our scenario. This extension of the time horizon forced us to also modify the time windows of the requests so that they could be served throughout the entire period. We employed the following procedure: given a request $(p, d)$ for a pickup node $p$ and delivery node $d$, associated with time windows $\left[e_{p}, l_{p}\right]$ and $\left[e_{d}, l_{d}\right]$, we shift the original time windows by

$$
\begin{aligned}
e_{p} & =e_{p}+R \\
l_{p} & =l_{p}+R \\
e_{d} & =e_{d}+R \\
l_{d} & =l_{d}+R
\end{aligned}
$$


where $R$ is a random integer in the interval [0,600]. In this way, the requests of the original instances are spread over the longer time horizon, while still taking into account their original values.

Service times of 5 minutes were added to every customer location (and transfer operation) since the original instances assumed a value of zero for them. In our case, they are directly related to the working hours.

Finally, following Sampaio et al. (2020) we assume that every unit of travel time equals 1 minute. Thus, all times in the instances are represented as minutes for simplicity.

\section{E.2. Graphs}

Figure 11: TDSP-IR instance characteristics according to the number of interdependencies.

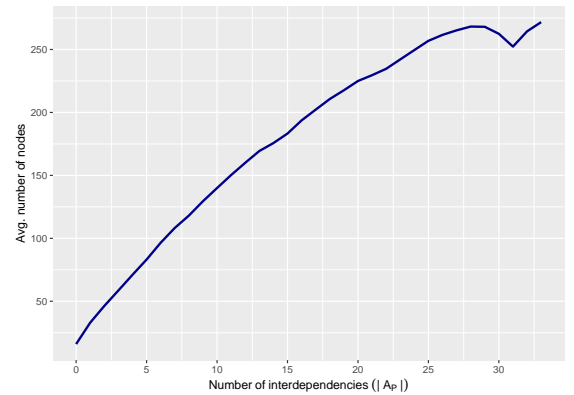

(a) Average number of nodes

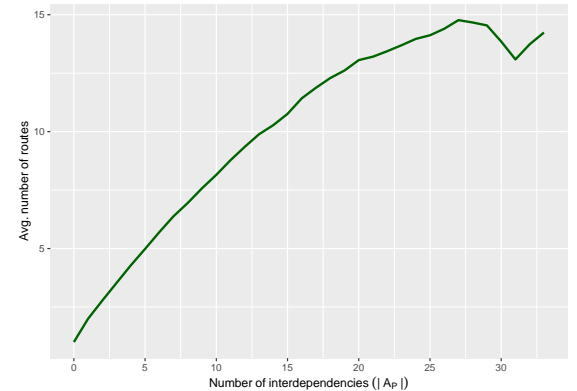

(b) Average number of routes

\section{E.3. Tables}

The tables in this appendix present detailed execution times for both the LPA and the MILP. Tables 8, 9 and 10 report results over all TDSP-IR instances and for maximum route durations of $M_{\text {dur }}=8 \mathrm{~h}, M_{\text {dur }}=10 \mathrm{~h}$ and $M_{\text {dur }}=13 \mathrm{~h}$, respectively. Similarly, Tables 11,12 and 13 report results only over the feasible TDSP-IR instances. For each table, we report the minimum, average (and standard deviation), and maximum recorded running times of the scheduling algorithms according to the number of interdependencies $\left|A_{P}\right|$. 
Table 8: Detailed runtimes over all instances for each algorithm $\left(M_{\mathrm{dur}}=8 \mathrm{~h}\right)$.

\begin{tabular}{cccrcccr} 
& \multicolumn{3}{c}{ LPA } & & & \multicolumn{3}{c}{ MILP } \\
\cline { 2 - 3 } \cline { 6 - 7 }$\left|A_{P}\right|$ & min & avg $\pm \mathrm{sd}$ & $\max$ & & min & avg $\pm \mathrm{sd}$ & $\max$ \\
\hline 0 & 0.00 & $0.01 \pm 0.01$ & 1.12 & & 0.12 & $0.49 \pm 0.18$ & 7.93 \\
1 & 0.00 & $0.01 \pm 0.01$ & 0.64 & & 0.19 & $0.81 \pm 0.20$ & 10.25 \\
2 & 0.00 & $0.01 \pm 0.01$ & 0.88 & & 0.24 & $1.08 \pm 0.29$ & 5.48 \\
3 & 0.00 & $0.01 \pm 0.02$ & 0.69 & & 0.30 & $1.32 \pm 0.35$ & 6.28 \\
4 & 0.00 & $0.01 \pm 0.02$ & 1.39 & & 0.36 & $1.57 \pm 0.41$ & 7.12 \\
5 & 0.00 & $0.01 \pm 0.03$ & 2.88 & & 0.41 & $1.82 \pm 0.49$ & 8.25 \\
6 & 0.00 & $0.01 \pm 0.04$ & 3.12 & & 0.50 & $2.03 \pm 0.54$ & 7.68 \\
7 & 0.00 & $0.02 \pm 0.05$ & 6.28 & & 0.49 & $2.25 \pm 0.59$ & 10.10 \\
8 & 0.00 & $0.02 \pm 0.08$ & 12.14 & & 0.59 & $2.47 \pm 0.65$ & 9.08 \\
9 & 0.00 & $0.02 \pm 0.10$ & 17.26 & & 0.63 & $2.71 \pm 0.72$ & 9.84 \\
10 & 0.00 & $0.03 \pm 0.27$ & 57.30 & & 0.67 & $2.92 \pm 0.77$ & 11.26 \\
11 & 0.00 & $0.04 \pm 0.76$ & 232.78 & & 0.72 & $3.13 \pm 0.82$ & 12.32 \\
12 & 0.00 & $0.05 \pm 0.45$ & 98.91 & & 0.72 & $3.31 \pm 0.86$ & 11.96 \\
13 & 0.00 & $0.05 \pm 0.66$ & 155.99 & & 0.78 & $3.50 \pm 0.92$ & 11.81 \\
14 & 0.00 & $0.06 \pm 0.37$ & 35.87 & & 0.83 & $3.72 \pm 1.01$ & 12.51 \\
15 & 0.00 & $0.08 \pm 0.68$ & 70.39 & & 0.87 & $3.94 \pm 1.09$ & 12.89 \\
16 & 0.00 & $0.11 \pm 1.03$ & 163.08 & & 0.96 & $4.13 \pm 1.13$ & 13.85 \\
17 & 0.00 & $0.12 \pm 1.23$ & 78.12 & & 1.00 & $4.28 \pm 1.15$ & 14.85 \\
18 & 0.00 & $0.11 \pm 1.56$ & 176.22 & & 1.09 & $4.52 \pm 1.17$ & 17.23 \\
19 & 0.00 & $0.15 \pm 2.42$ & 279.16 & & 1.16 & $4.70 \pm 1.23$ & 15.10 \\
20 & 0.00 & $0.13 \pm 1.36$ & 396.92 & & 1.17 & $4.84 \pm 1.31$ & 15.85 \\
21 & 0.00 & $0.23 \pm 2.88$ & 241.81 & & 1.23 & $4.98 \pm 1.39$ & 15.61 \\
22 & 0.00 & $0.42 \pm 7.36$ & 636.73 & & 1.29 & $5.03 \pm 1.35$ & 15.58 \\
23 & 0.00 & $0.51 \pm 8.40$ & 1202.17 & & 1.35 & $5.17 \pm 1.32$ & 14.69 \\
24 & 0.00 & $0.37 \pm 10.70$ & 997.08 & & 1.33 & $5.21 \pm 1.41$ & 16.91 \\
25 & 0.00 & $0.27 \pm 2.56$ & 133.44 & & 1.34 & $5.31 \pm 1.37$ & 15.20 \\
26 & 0.00 & $0.22 \pm 2.36$ & 159.08 & & 1.42 & $5.31 \pm 1.44$ & 13.81 \\
27 & 0.00 & $0.66 \pm 1.79$ & 149.58 & & 1.48 & $5.35 \pm 1.29$ & 14.00 \\
28 & 0.00 & $0.83 \pm 0.84$ & 46.73 & & 1.55 & $5.49 \pm 1.31$ & 12.80 \\
29 & 0.00 & $0.84 \pm 0.25$ & 2.86 & & 1.77 & $5.58 \pm 1.07$ & 12.91 \\
30 & 0.00 & $1.00 \pm 0.25$ & 3.13 & & 1.84 & $5.72 \pm 0.94$ & 11.47 \\
31 & 0.00 & $1.18 \pm 0.30$ & 2.89 & & 1.88 & $6.09 \pm 0.97$ & 11.70 \\
32 & 0.00 & $1.25 \pm 0.23$ & 3.06 & & 1.98 & $6.11 \pm 0.77$ & 11.39 \\
33 & 0.00 & $1.09 \pm 0.24$ & 1.46 & & 2.06 & $6.17 \pm 0.79$ & 10.55 \\
34 & 0.00 & $1.06 \pm 0.17$ & 1.25 & & 5.89 & $6.26 \pm 0.54$ & 9.77 \\
\hline & & & & & & &
\end{tabular}

Table 9: Detailed runtimes over all instances for each algorithm $\left(M_{\mathrm{dur}}=10 \mathrm{~h}\right)$.

\begin{tabular}{|c|c|c|c|c|c|c|}
\hline \multirow[b]{2}{*}{$\left|A_{P}\right|$} & \multicolumn{3}{|c|}{ LPA } & \multicolumn{3}{|c|}{ MILP } \\
\hline & $\min$ & $\operatorname{avg} \pm \mathrm{sd}$ & $\max$ & $\min$ & $\operatorname{avg} \pm \mathrm{sd}$ & $\max$ \\
\hline 0 & 0.00 & $0.01 \pm 0.01$ & 1.55 & 0.14 & $0.62 \pm 0.38$ & 25.87 \\
\hline 1 & 0.00 & $0.01 \pm 0.02$ & 1.61 & 0.20 & $1.10 \pm 0.61$ & 25.95 \\
\hline 2 & 0.00 & $0.02 \pm 0.03$ & 1.60 & 0.27 & $1.46 \pm 0.82$ & 27.88 \\
\hline 3 & 0.00 & $0.03 \pm 0.04$ & 1.05 & 0.30 & $1.77 \pm 1.00$ & 27.13 \\
\hline 4 & 0.00 & $0.04 \pm 0.05$ & 1.90 & 0.41 & $2.22 \pm 1.28$ & 29.56 \\
\hline 5 & 0.00 & $0.05 \pm 0.07$ & 2.45 & 0.46 & $2.54 \pm 1.42$ & 32.47 \\
\hline 6 & 0.00 & $0.06 \pm 0.11$ & 7.14 & 0.49 & $2.84 \pm 1.62$ & 30.52 \\
\hline 7 & 0.00 & $0.08 \pm 0.16$ & 19.08 & 0.63 & $3.23 \pm 1.84$ & 66.65 \\
\hline 8 & 0.00 & $0.11 \pm 0.33$ & 39.02 & 0.65 & $3.55 \pm 2.06$ & 32.71 \\
\hline 9 & 0.00 & $0.13 \pm 0.36$ & 30.58 & 0.71 & $3.88 \pm 2.24$ & 35.63 \\
\hline 10 & 0.00 & $0.15 \pm 0.46$ & 34.43 & 0.75 & $4.07 \pm 2.37$ & 33.29 \\
\hline 11 & 0.00 & $0.17 \pm 0.56$ & 74.56 & 0.78 & $4.32 \pm 2.48$ & 35.45 \\
\hline 12 & 0.00 & $0.22 \pm 1.12$ & 119.38 & 0.87 & $4.55 \pm 2.59$ & 37.96 \\
\hline 13 & 0.00 & $0.28 \pm 1.50$ & 186.71 & 0.93 & $4.84 \pm 2.74$ & 35.39 \\
\hline 14 & 0.00 & $0.30 \pm 1.47$ & 141.59 & 0.81 & $5.04 \pm 2.78$ & 53.60 \\
\hline 15 & 0.00 & $0.35 \pm 1.75$ & 183.17 & 0.97 & $5.24 \pm 2.90$ & 41.79 \\
\hline 16 & 0.00 & $0.40 \pm 1.92$ & 247.39 & 1.02 & $5.46 \pm 3.02$ & 52.75 \\
\hline 17 & 0.00 & $0.47 \pm 3.53$ & 701.92 & 1.08 & $5.61 \pm 3.09$ & 39.60 \\
\hline 18 & 0.00 & $0.80 \pm 10.00$ & 1993.29 & 1.07 & $5.84 \pm 3.20$ & 41.61 \\
\hline 19 & 0.00 & $0.94 \pm 9.13$ & 1294.59 & 1.08 & $5.99 \pm 3.24$ & 39.04 \\
\hline 20 & 0.00 & $0.74 \pm 4.75$ & 395.94 & 1.25 & $6.22 \pm 3.32$ & 38.58 \\
\hline 21 & 0.00 & $0.97 \pm 7.55$ & 563.25 & 1.34 & $6.30 \pm 3.39$ & 38.63 \\
\hline 22 & 0.00 & $0.92 \pm 6.56$ & 781.82 & 1.37 & $6.38 \pm 3.38$ & 34.62 \\
\hline 23 & 0.00 & $1.01 \pm 11.32$ & 1514.07 & 1.39 & $6.43 \pm 3.45$ & 37.91 \\
\hline 24 & 0.00 & $0.89 \pm 6.58$ & 416.41 & 1.42 & $6.51 \pm 3.45$ & 30.25 \\
\hline 25 & 0.00 & $1.17 \pm 8.17$ & 321.05 & 1.47 & $6.64 \pm 3.38$ & 39.08 \\
\hline 26 & 0.00 & $0.95 \pm 6.56$ & 263.35 & 1.52 & $6.94 \pm 3.57$ & 38.46 \\
\hline 27 & 0.00 & $1.81 \pm 10.93$ & 500.18 & 1.55 & $7.24 \pm 3.54$ & 29.91 \\
\hline 28 & 0.00 & $1.29 \pm 8.84$ & 599.67 & 1.62 & $7.40 \pm 3.51$ & 35.81 \\
\hline 29 & 0.00 & $3.06 \pm 21.02$ & 814.51 & 1.71 & $7.66 \pm 3.86$ & 39.63 \\
\hline 30 & 0.00 & $1.92 \pm 12.13$ & 318.29 & 1.79 & $7.32 \pm 3.31$ & 39.94 \\
\hline 31 & 0.00 & $6.20 \pm 26.94$ & 624.27 & 1.80 & $7.22 \pm 3.42$ & 17.41 \\
\hline 32 & 0.00 & $1.92 \pm 7.47$ & 196.63 & 1.92 & $8.34 \pm 4.10$ & 24.21 \\
\hline 33 & 0.00 & $0.72 \pm 3.17$ & 43.17 & 2.03 & $7.06 \pm 2.77$ & 15.96 \\
\hline 34 & 0.00 & $1.50 \pm 5.18$ & 41.19 & 2.11 & $7.51 \pm 2.95$ & 16.11 \\
\hline
\end{tabular}


Table 10: Detailed runtimes over all instances for each algorithm $\left(M_{\mathrm{dur}}=13 \mathrm{~h}\right)$.

\begin{tabular}{|c|c|c|c|c|c|c|}
\hline \multirow[b]{2}{*}{$\left|A_{P}\right|$} & \multicolumn{3}{|c|}{ LPA } & \multicolumn{3}{|c|}{ MILP } \\
\hline & $\min$ & $\operatorname{avg} \pm \mathrm{sd}$ & $\max$ & $\min$ & $\operatorname{avg} \pm \mathrm{sd}$ & $\max$ \\
\hline 0 & 0.00 & $0.01 \pm 0.01$ & 1.62 & 0.15 & $0.56 \pm 0.45$ & 22.43 \\
\hline 1 & 0.00 & $0.03 \pm 0.02$ & 2.01 & 0.20 & $1.32 \pm 0.79$ & 21.94 \\
\hline 2 & 0.00 & $0.04 \pm 0.03$ & 1.07 & 0.28 & $1.82 \pm 1.04$ & 27.16 \\
\hline 3 & 0.00 & $0.06 \pm 0.04$ & 3.43 & 0.37 & $2.44 \pm 1.42$ & 27.52 \\
\hline 4 & 0.00 & $0.07 \pm 0.06$ & 0.68 & 0.41 & $2.78 \pm 1.64$ & 29.01 \\
\hline 5 & 0.00 & $0.09 \pm 0.08$ & 1.98 & 0.42 & $3.22 \pm 1.83$ & 28.15 \\
\hline 6 & 0.00 & $0.13 \pm 0.11$ & 2.49 & 0.45 & $3.73 \pm 2.13$ & 30.74 \\
\hline 7 & 0.00 & $0.17 \pm 0.15$ & 5.60 & 0.61 & $4.25 \pm 2.40$ & 32.07 \\
\hline 8 & 0.00 & $0.21 \pm 0.21$ & 13.28 & 0.67 & $4.68 \pm 2.61$ & 32.87 \\
\hline 9 & 0.00 & $0.25 \pm 0.26$ & 18.67 & 0.78 & $5.11 \pm 2.74$ & 34.03 \\
\hline 10 & 0.00 & $0.29 \pm 0.29$ & 18.55 & 0.76 & $5.48 \pm 2.93$ & 33.77 \\
\hline 11 & 0.00 & $0.34 \pm 0.38$ & 22.83 & 0.85 & $5.83 \pm 3.06$ & 35.35 \\
\hline 12 & 0.00 & $0.41 \pm 0.65$ & 28.49 & 0.96 & $6.11 \pm 3.20$ & 34.06 \\
\hline 13 & 0.00 & $0.47 \pm 0.99$ & 77.45 & 1.03 & $6.44 \pm 3.28$ & 35.45 \\
\hline 14 & 0.00 & $0.53 \pm 1.17$ & 79.47 & 0.95 & $6.69 \pm 3.46$ & 36.87 \\
\hline 15 & 0.00 & $0.62 \pm 1.64$ & 84.49 & 1.07 & $7.13 \pm 3.68$ & 37.36 \\
\hline 16 & 0.00 & $0.63 \pm 1.57$ & 116.94 & 1.07 & $7.16 \pm 3.63$ & 36.97 \\
\hline 17 & 0.00 & $0.73 \pm 1.83$ & 143.59 & 1.10 & $7.45 \pm 3.76$ & 37.89 \\
\hline 18 & 0.00 & $0.93 \pm 2.68$ & 90.30 & 1.15 & $7.88 \pm 3.89$ & 37.93 \\
\hline 19 & 0.00 & $0.99 \pm 2.61$ & 258.38 & 1.23 & $8.16 \pm 4.01$ & 38.55 \\
\hline 20 & 0.00 & $1.13 \pm 3.25$ & 201.66 & 1.27 & $8.50 \pm 4.08$ & 38.98 \\
\hline 21 & 0.00 & $1.30 \pm 4.46$ & 258.34 & 1.28 & $8.59 \pm 4.10$ & 38.18 \\
\hline 22 & 0.00 & $1.46 \pm 6.59$ & 369.20 & 1.40 & $8.42 \pm 4.13$ & 37.90 \\
\hline 23 & 0.00 & $2.24 \pm 11.12$ & 399.18 & 1.48 & $8.66 \pm 4.21$ & 38.33 \\
\hline 24 & 0.00 & $2.96 \pm 16.62$ & 414.53 & 1.49 & $9.03 \pm 4.16$ & 38.50 \\
\hline 25 & 0.00 & $3.21 \pm 21.10$ & 1150.30 & 1.47 & $8.73 \pm 3.88$ & 34.96 \\
\hline 26 & 0.00 & $3.55 \pm 35.45$ & 1126.43 & 1.48 & $8.91 \pm 3.74$ & 33.25 \\
\hline 27 & 0.00 & $3.56 \pm 21.23$ & 689.95 & 1.58 & $9.06 \pm 3.62$ & 19.21 \\
\hline 28 & 0.00 & $6.17 \pm 33.18$ & 526.44 & 1.83 & $8.67 \pm 3.33$ & 34.24 \\
\hline 29 & 0.00 & $9.38 \pm 59.35$ & 958.84 & 1.87 & $8.14 \pm 3.70$ & 33.57 \\
\hline 30 & 0.00 & $7.07 \pm 52.51$ & 732.06 & 1.94 & $8.17 \pm 3.58$ & 15.83 \\
\hline 31 & 0.00 & $1.05 \pm 1.71$ & 12.47 & 1.91 & $9.00 \pm 3.17$ & 14.83 \\
\hline 32 & 0.79 & $0.87 \pm 0.09$ & 0.99 & 6.20 & $11.75 \pm 3.80$ & 14.83 \\
\hline
\end{tabular}

Table 11: Detailed runtimes over feasible instances for each algorithm $\left(M_{\mathrm{dur}}=8 \mathrm{~h}\right)$.

\begin{tabular}{|c|c|c|c|c|c|c|}
\hline \multirow[b]{2}{*}{$\left|A_{P}\right|$} & \multicolumn{3}{|c|}{ LPA } & \multicolumn{3}{|c|}{ MILP } \\
\hline & $\min$ & $\operatorname{avg} \pm \mathrm{sd}$ & $\max$ & $\min$ & avg \pm sd & $\max$ \\
\hline 0 & 0.01 & $0.01 \pm 0.01$ & 1.12 & 0.23 & $0.54 \pm 0.27$ & 6.41 \\
\hline 1 & 0.01 & $0.03 \pm 0.01$ & 0.43 & 0.36 & $1.17 \pm 0.54$ & 5.58 \\
\hline 2 & 0.01 & $0.05 \pm 0.02$ & 0.74 & 0.39 & $1.85 \pm 0.62$ & 5.48 \\
\hline 3 & 0.02 & $0.07 \pm 0.02$ & 0.69 & 0.46 & $2.40 \pm 0.63$ & 5.77 \\
\hline 4 & 0.02 & $0.09 \pm 0.03$ & 0.57 & 0.60 & $2.94 \pm 0.74$ & 6.55 \\
\hline 5 & 0.02 & $0.11 \pm 0.03$ & 0.99 & 0.71 & $3.47 \pm 0.82$ & 8.01 \\
\hline 6 & 0.04 & $0.13 \pm 0.04$ & 0.99 & 0.95 & $3.85 \pm 0.86$ & 7.56 \\
\hline 7 & 0.03 & $0.15 \pm 0.05$ & 0.87 & 1.03 & $4.34 \pm 0.94$ & 7.79 \\
\hline 8 & 0.04 & $0.18 \pm 0.07$ & 2.07 & 1.85 & $4.83 \pm 0.97$ & 9.08 \\
\hline 9 & 0.05 & $0.21 \pm 0.07$ & 1.91 & 2.47 & $5.23 \pm 1.01$ & 9.84 \\
\hline 10 & 0.06 & $0.25 \pm 0.10$ & 6.00 & 2.70 & $5.65 \pm 1.09$ & 11.26 \\
\hline 11 & 0.07 & $0.29 \pm 0.32$ & 26.33 & 3.05 & $6.03 \pm 1.07$ & 12.32 \\
\hline 12 & 0.07 & $0.34 \pm 0.17$ & 7.01 & 3.49 & $6.46 \pm 1.16$ & 11.96 \\
\hline 13 & 0.09 & $0.42 \pm 0.53$ & 21.50 & 3.56 & $6.83 \pm 1.23$ & 11.81 \\
\hline 14 & 0.09 & $0.48 \pm 0.46$ & 14.56 & 4.02 & $7.50 \pm 1.42$ & 12.51 \\
\hline 15 & 0.13 & $0.50 \pm 0.31$ & 19.82 & 4.35 & $7.91 \pm 1.34$ & 12.89 \\
\hline 16 & 0.18 & $0.57 \pm 0.31$ & 7.35 & 4.20 & $8.26 \pm 1.31$ & 13.85 \\
\hline 17 & 0.24 & $0.61 \pm 0.36$ & 12.80 & 4.75 & $8.54 \pm 1.46$ & 14.85 \\
\hline 18 & 0.29 & $0.64 \pm 0.37$ & 15.09 & 4.74 & $8.71 \pm 1.47$ & 17.23 \\
\hline 19 & 0.31 & $0.67 \pm 0.47$ & 24.14 & 5.23 & $9.13 \pm 1.38$ & 15.10 \\
\hline 20 & 0.30 & $0.69 \pm 0.39$ & 9.06 & 5.96 & $9.43 \pm 1.54$ & 15.85 \\
\hline 21 & 0.35 & $0.77 \pm 0.74$ & 22.31 & 6.07 & $9.89 \pm 1.44$ & 15.61 \\
\hline 22 & 0.37 & $0.79 \pm 0.68$ & 18.85 & 6.53 & $9.92 \pm 1.59$ & 15.58 \\
\hline 23 & 0.44 & $1.04 \pm 1.53$ & 22.58 & 6.63 & $10.39 \pm 1.63$ & 14.69 \\
\hline 24 & 0.46 & $0.90 \pm 1.11$ & 35.09 & 6.43 & $10.12 \pm 1.59$ & 16.91 \\
\hline 25 & 0.49 & $1.42 \pm 2.75$ & 28.55 & 6.43 & $10.22 \pm 1.33$ & 15.20 \\
\hline 26 & 0.55 & $1.21 \pm 2.71$ & 50.98 & 7.34 & $10.27 \pm 1.04$ & 13.81 \\
\hline 27 & 0.55 & $0.92 \pm 1.30$ & 22.80 & 8.11 & $9.66 \pm 1.13$ & 14.00 \\
\hline 28 & 0.61 & $0.84 \pm 0.17$ & 2.79 & 7.48 & $10.01 \pm 0.84$ & 12.80 \\
\hline 29 & 0.65 & $0.94 \pm 0.12$ & 1.49 & 7.77 & $10.42 \pm 0.99$ & 12.91 \\
\hline 30 & 0.89 & $0.99 \pm 0.06$ & 1.09 & 9.16 & $10.37 \pm 0.61$ & 11.47 \\
\hline 31 & 0.91 & $1.15 \pm 0.08$ & 1.37 & 9.22 & $10.46 \pm 0.48$ & 11.70 \\
\hline 32 & 0.94 & $1.18 \pm 0.11$ & 1.42 & 9.05 & $10.09 \pm 0.71$ & 11.39 \\
\hline 33 & 0.95 & $1.08 \pm 0.09$ & 1.30 & 8.99 & $9.41 \pm 0.42$ & 10.55 \\
\hline 34 & 1.08 & $1.17 \pm 0.09$ & 1.25 & 9.32 & $9.48 \pm 0.25$ & 9.77 \\
\hline
\end{tabular}


Table 12: Detailed runtimes over feasible instances for each algorithm $\left(M_{\mathrm{dur}}=10 \mathrm{~h}\right)$.

\begin{tabular}{cccccccc} 
& \multicolumn{3}{c}{ LPA } & & & & \\
\cline { 7 - 8 }$\left|A_{P}\right|$ & min & avg $\pm \mathrm{sd}$ & $\max$ & & $\min$ & avg $\pm \mathrm{sd}$ & $\max$ \\
\hline 0 & 0.01 & $0.02 \pm 0.01$ & 1.55 & & 0.24 & $0.64 \pm 0.55$ & 25.87 \\
1 & 0.01 & $0.05 \pm 0.02$ & 1.61 & & 0.37 & $1.95 \pm 0.87$ & 25.95 \\
2 & 0.01 & $0.07 \pm 0.02$ & 1.60 & & 0.40 & $2.67 \pm 0.98$ & 27.88 \\
3 & 0.02 & $0.09 \pm 0.03$ & 0.97 & & 0.54 & $3.44 \pm 1.09$ & 27.13 \\
4 & 0.03 & $0.12 \pm 0.04$ & 1.90 & & 0.66 & $4.24 \pm 1.31$ & 29.56 \\
5 & 0.04 & $0.15 \pm 0.06$ & 2.45 & & 1.03 & $4.81 \pm 1.39$ & 32.47 \\
6 & 0.05 & $0.19 \pm 0.09$ & 2.12 & & 1.09 & $5.53 \pm 1.50$ & 30.52 \\
7 & 0.05 & $0.24 \pm 0.11$ & 4.84 & & 1.95 & $6.29 \pm 1.63$ & 30.85 \\
8 & 0.05 & $0.30 \pm 0.15$ & 6.52 & & 2.09 & $7.00 \pm 1.74$ & 32.71 \\
9 & 0.05 & $0.35 \pm 0.17$ & 7.97 & & 2.57 & $7.69 \pm 1.79$ & 35.63 \\
10 & 0.05 & $0.39 \pm 0.18$ & 7.57 & & 2.96 & $8.16 \pm 1.88$ & 33.29 \\
11 & 0.09 & $0.45 \pm 0.25$ & 17.45 & & 3.28 & $8.56 \pm 1.92$ & 35.45 \\
12 & 0.11 & $0.50 \pm 0.28$ & 26.89 & & 3.49 & $9.02 \pm 1.93$ & 37.96 \\
13 & 0.12 & $0.59 \pm 0.50$ & 19.00 & & 3.64 & $9.49 \pm 1.90$ & 35.39 \\
14 & 0.11 & $0.62 \pm 0.64$ & 75.03 & & 3.52 & $9.71 \pm 1.93$ & 35.82 \\
15 & 0.12 & $0.69 \pm 0.65$ & 42.45 & & 3.76 & $10.16 \pm 2.02$ & 41.79 \\
16 & 0.13 & $0.74 \pm 0.73$ & 56.32 & & 4.11 & $10.39 \pm 2.05$ & 40.69 \\
17 & 0.15 & $0.82 \pm 1.09$ & 47.25 & & 4.33 & $10.79 \pm 2.09$ & 39.60 \\
18 & 0.24 & $0.91 \pm 2.28$ & 182.04 & & 5.38 & $11.11 \pm 2.18$ & 41.61 \\
19 & 0.23 & $1.14 \pm 3.65$ & 135.63 & & 4.80 & $11.23 \pm 2.11$ & 39.04 \\
20 & 0.26 & $1.01 \pm 2.48$ & 225.64 & & 5.38 & $11.54 \pm 2.07$ & 38.58 \\
21 & 0.28 & $1.16 \pm 2.36$ & 97.66 & & 6.44 & $11.73 \pm 1.89$ & 38.63 \\
22 & 0.31 & $1.25 \pm 2.73$ & 105.53 & & 6.56 & $12.07 \pm 1.97$ & 34.62 \\
23 & 0.32 & $1.21 \pm 2.09$ & 71.89 & & 6.54 & $12.25 \pm 2.00$ & 37.91 \\
24 & 0.35 & $1.27 \pm 2.21$ & 93.63 & & 6.64 & $12.41 \pm 2.15$ & 24.59 \\
25 & 0.43 & $1.08 \pm 1.07$ & 35.25 & & 7.07 & $12.42 \pm 2.52$ & 39.08 \\
26 & 0.43 & $1.39 \pm 1.96$ & 34.30 & & 7.99 & $13.27 \pm 2.72$ & 38.46 \\
27 & 0.46 & $1.32 \pm 3.36$ & 80.57 & & 7.56 & $13.03 \pm 2.08$ & 23.61 \\
28 & 0.53 & $1.31 \pm 2.51$ & 40.84 & & 8.49 & $13.44 \pm 1.84$ & 35.81 \\
29 & 0.62 & $1.38 \pm 3.97$ & 84.23 & & 9.50 & $14.13 \pm 2.01$ & 39.63 \\
30 & 0.68 & $1.38 \pm 2.39$ & 42.78 & & 9.11 & $13.87 \pm 1.61$ & 39.94 \\
31 & 0.70 & $1.49 \pm 2.66$ & 42.15 & & 9.48 & $14.50 \pm 1.58$ & 17.41 \\
32 & 0.79 & $1.80 \pm 4.92$ & 57.72 & & 12.02 & $15.59 \pm 1.34$ & 24.21 \\
33 & 0.87 & $1.31 \pm 2.14$ & 16.15 & & 12.16 & $14.03 \pm 0.65$ & 15.96 \\
34 & 0.88 & $1.16 \pm 1.48$ & 15.84 & & 12.59 & $14.13 \pm 0.83$ & 16.11 \\
\hline & & & & & &
\end{tabular}

Table 13: Detailed runtimes over feasible instances for each algorithm $\left(M_{\mathrm{dur}}=13 \mathrm{~h}\right)$.

\begin{tabular}{cccrrrrr}
\hline & \multicolumn{3}{c}{ LPA } & & \multicolumn{3}{c}{ MILP } \\
\cline { 2 - 3 } \cline { 5 - 6 }$\left|A_{P}\right|$ & min & avg $\pm \mathrm{sd}$ & $\max$ & & $\min$ & avg $\pm \mathrm{sd}$ & $\max$ \\
\hline 0 & 0.00 & $0.01 \pm 0.01$ & 1.62 & & 0.15 & $0.56 \pm 0.45$ & 22.43 \\
1 & 0.00 & $0.03 \pm 0.02$ & 2.01 & & 0.20 & $1.32 \pm 0.79$ & 21.94 \\
2 & 0.00 & $0.04 \pm 0.03$ & 1.07 & & 0.28 & $1.82 \pm 1.04$ & 27.16 \\
3 & 0.00 & $0.06 \pm 0.04$ & 3.43 & & 0.37 & $2.44 \pm 1.42$ & 27.52 \\
4 & 0.00 & $0.07 \pm 0.06$ & 0.68 & & 0.41 & $2.78 \pm 1.64$ & 29.01 \\
5 & 0.00 & $0.09 \pm 0.08$ & 1.98 & & 0.42 & $3.22 \pm 1.83$ & 28.15 \\
6 & 0.00 & $0.13 \pm 0.11$ & 2.49 & & 0.45 & $3.73 \pm 2.13$ & 30.74 \\
7 & 0.00 & $0.17 \pm 0.15$ & 5.60 & & 0.61 & $4.25 \pm 2.40$ & 32.07 \\
8 & 0.00 & $0.21 \pm 0.21$ & 13.28 & & 0.67 & $4.68 \pm 2.61$ & 32.87 \\
9 & 0.00 & $0.25 \pm 0.26$ & 18.67 & & 0.78 & $5.11 \pm 2.74$ & 34.03 \\
10 & 0.00 & $0.29 \pm 0.29$ & 18.55 & & 0.76 & $5.48 \pm 2.93$ & 33.77 \\
11 & 0.00 & $0.34 \pm 0.38$ & 22.83 & & 0.85 & $5.83 \pm 3.06$ & 35.35 \\
12 & 0.00 & $0.41 \pm 0.65$ & 28.49 & & 0.96 & $6.11 \pm 3.20$ & 34.06 \\
13 & 0.00 & $0.47 \pm 0.99$ & 77.45 & & 1.03 & $6.44 \pm 3.28$ & 35.45 \\
14 & 0.00 & $0.53 \pm 1.17$ & 79.47 & & 0.95 & $6.69 \pm 3.46$ & 36.87 \\
15 & 0.00 & $0.62 \pm 1.64$ & 84.49 & & 1.07 & $7.13 \pm 3.68$ & 37.36 \\
16 & 0.00 & $0.63 \pm 1.57$ & 116.94 & & 1.07 & $7.16 \pm 3.63$ & 36.97 \\
17 & 0.00 & $0.73 \pm 1.83$ & 143.59 & & 1.10 & $7.45 \pm 3.76$ & 37.89 \\
18 & 0.00 & $0.93 \pm 2.68$ & 90.30 & & 1.15 & $7.88 \pm 3.89$ & 37.93 \\
19 & 0.00 & $0.99 \pm 2.61$ & 258.38 & & 1.23 & $8.16 \pm 4.01$ & 38.55 \\
20 & 0.00 & $1.13 \pm 3.25$ & 201.66 & & 1.27 & $8.50 \pm 4.08$ & 38.98 \\
21 & 0.00 & $1.30 \pm 4.46$ & 258.34 & & 1.28 & $8.59 \pm 4.10$ & 38.18 \\
22 & 0.00 & $1.46 \pm 6.59$ & 369.20 & & 1.40 & $8.42 \pm 4.13$ & 37.90 \\
23 & 0.00 & $2.24 \pm 11.12$ & 399.18 & & 1.48 & $8.66 \pm 4.21$ & 38.33 \\
24 & 0.00 & $2.96 \pm 16.62$ & 414.53 & & 1.49 & $9.03 \pm 4.16$ & 38.50 \\
25 & 0.00 & $3.21 \pm 21.10$ & 1150.30 & & 1.47 & $8.73 \pm 3.88$ & 34.96 \\
26 & 0.00 & $3.55 \pm 35.45$ & 1126.43 & & 1.48 & $8.91 \pm 3.74$ & 33.25 \\
27 & 0.00 & $3.56 \pm 21.23$ & 689.95 & & 1.58 & $9.06 \pm 3.62$ & 19.21 \\
28 & 0.00 & $6.17 \pm 33.18$ & 526.44 & & 1.83 & $8.67 \pm 3.33$ & 34.24 \\
29 & 0.00 & $9.38 \pm 59.35$ & 958.84 & & 1.87 & $8.14 \pm 3.70$ & 33.57 \\
30 & 0.00 & $7.07 \pm 52.51$ & 732.06 & & 1.94 & $8.17 \pm 3.58$ & 15.83 \\
31 & 0.00 & $1.05 \pm 1.71$ & 12.47 & & 1.91 & $9.00 \pm 3.17$ & 14.83 \\
32 & 0.79 & $0.87 \pm 0.09$ & 0.99 & & 6.20 & $11.75 \pm 3.80$ & 14.83 \\
\hline & & & & & &
\end{tabular}

